\title{
RIS-Enhanced WPCNs: Joint Radio Resource Allocation and Passive Beamforming Optimization
}

\author{
Yongjun Xu, Senior Member, IEEE, Zhengnian Gao, Zhengqiang Wang, Member, IEEE, Chongwen \\ Huang, Member, IEEE, Zhaohui Yang, Member, IEEE, and Chau Yuen, Fellow, IEEE
}

\begin{abstract}
Wireless-powered communication and reconfigurable intelligent surface (RIS) can complement each other for increasing energy utilization and spectrum efficiency by reconfiguring the surrounding radio environment, however, which has not been sufficiently studied by the existing works. In this paper, we propose a joint radio resource and passive beamforming optimization scheme for a downlink RIS-assisted wireless-powered communication network with a harvest-thentransmit protocol to improve system energy efficiency (EE). In the considered model, the single-antenna wireless devices (WDs) harvest wireless energy from a multi-antenna dedicated power station (PS) through the RIS in the downlink and transmit their independent information to a single-antenna receiver in the uplink by a time-division-multiple-access mode. Our goal is to maximize the total $\mathrm{EE}$ of all WDs. To make full use of the beamforming gain provided by both the PS and the RIS, we jointly optimize the active beamforming of the PS and the passive beamforming of the RIS. To deal with the challenging nonconvex optimization problem with multiple coupled variables, we first consider fixing the passive beamforming, and converting the remaining radio resource allocation problem into an equivalent convex problem which is solved by using Lagrange dual theory. Then, we fix the optimized resource allocation parameters and optimize the passive beamforming of the RIS by using a semidefinite programming method. Simulation results demonstrate that the proposed algorithm achieves higher EE compared to the conventional schemes.
\end{abstract}

Index Terms-Reconfigurable intelligent surface, wirelesspowered communication, energy efficiency, passive beamforming,

This work was supported by the National Natural Science Foundation of China (61601071, 62071078), the Natural Science Foundation of Chongqing (cstc2019jcyj-xfkxX0002, cstc2019jcyj-msxmX0666), Open Funding of Shaanxi Key Laboratory of Information Communication Network and Security (ICNS201904), and the Fundamental Research Funds for the Central Universities ( 2021FZZX001-21). (Corresponding authors: Yongjun Xu.)

Y. $\mathrm{Xu}$ is with the School of Communication and Information Engineering, Chongqing Key Laboratory of Mobile Communications Technology, Chongqing University of Posts and Telecommunications, Chongqing 400065, China, and also with Shaanxi Key Laboratory of Information Communication Network and Security, Xian University of Posts \& Telecommunications, Xian, Shaanxi 710121, China (e-mail: xuyj@cqupt.edu.cn).

$Z$. Gao and Z. Wang are with the Chongqing University of Posts and Telecommunications, Chongqing 400065, China (e-mails: S190131173@stu.cqupt.edu.cn; wangzq@cqupt.edu.cn).

C. Huang is with College of Information Science and Electronic Engineering, Zhejiang University, Hangzhou 310027, China, and with Internationa Joint Innovation Center, Zhejiang University, Haining 314400, China, and also with Zhejiang Provincial Key Laboratory of Info. Proc., Commun. \& Netw. (IPCAN), Hangzhou 310027, China. (e-mail: chongwenhuang@zju.edu.cn).

Z. Yang is with the Centre for Telecommunications Research, Departmen of Engineering, King's College London, London WC2R 2LS, U.K. (e-mail yang.zhaohui@kcl.ac.uk).

C. Yuen is with the Engineering Product Development (EPD) Pillar, Singapore University of Technology and Design, Singapore 487372. (email:yuenchau@sutd.edu.sg). radio resource allocation.

\section{INTRODUCTION}

W ITH the rapid development of the fifth-generation $(5 \mathrm{G})$ communication, tens of billions of intelligent terminals are promising to be connected in wireless communication networks, which inevitably cause the explosion of data transmission and different service requests. Thus, the demand for ubiquitous connectivity of wireless devices (WDs) and high network capacity boosts many new techniques, such as massive multiple-input multiple-output (MIMO), cloud computing, cognitive networks, heterogeneous networks, etc [1][4]. Although these advanced communication technologies can improve spectrum efficiency and satisfy different communication requirements of diverse radio environments, they also induce high energy consumption and hardware cost.

Currently, wireless-powered communication networks (WPCNs) have been proposed to address this problem by using the dedicated wireless energy to charge the energy-limited devices [5]-[7]. Compared with the traditional battery-powered strategy, the WPCNs can provide suitable energy and achieve flexible wireless energy transfer in a low-cost operation way, such as wireless sensor networks [8], device-to-device (D2D) communication networks [9], wireless body area networks [40], etc. As a result, many scholars have focused on the resource allocation (RA) problems in WPCNs to improve system capacity, energy efficiency (EE), and prolong the lifetime of WDs.

In particular, the authors in [11] proposed an iteration-based time allocation algorithm to maximize the total throughput of multiple distributed WDs under the maximum transmission time constraint for a multiuser WPCN with a harvest-thentransmit (HTT) protocol, where all distributed WDs harvested the wireless energy by a hybrid access point (HAP) in the downlink and then sent their independent information to the HAP in the uplink by a time-division-multiple-access (TDMA) mode. The work in [12] considered a dual-hop WPCN with a full-duplex HAP and maximized the sum throughput of the uplink transmission under both amplify forward and decode forward modes. However, the above works [11] and [12] only considered the time allocation and ignored the power allocation of each WD. In [13], the authors studied the sum-throughput maximization problem for a relay-based wireless-powered uplink cellular network by jointly optimizing information transmission time and the transmit power of each WD. Besides, the throughput maximization RA problems for WPCNs were investigated in various scenarios, e.g., unmanned 
aerial vehicle (UAV)-enabled networks [14]-[16], ambient backscatter communication networks [17] and cognitive radio networks [18]. To further improve the uplink transmission quality of WDs with limited energy storage, the authors in [19] investigated the joint optimization problem of energy resource and time resource to maximize the sum rate of WDs in a WPCN with energy storage constraints. However, the improvement of system capacity is limited due to low energy conservation efficiency. In order to deal with this issue, MIMO technique was further employed in WPCNs in [20], where the authors studied a sum-throughput maximization RA problem by joint designing time allocation and energy beamforming vectors. To improve the fairness of WDs, the authors in [21] investigated a max-min throughput RA problem in a multiuser WPCN, where a multi-antenna HAP provided the energy signals to WDs by an active beamforming way. Furthermore, the weighted sum-rate maximization problems were studied in [22], [23] by optimizing energy harvesting (EH) time and transmit power, where the energy beamforming vector was also considered in [23]. In order to balance transmission rate and power consumption, the EE-based maximization problems were addressed in [24], [25]. Although we notice that the above works [11]-[14], [17]-[25] have optimized the data transmission time or the EH time and the beamforming vector of the base station (BS), the low energy transfer efficiency caused by the obstacles and time-varying channel fading is still a fundamental performance bottleneck of WPCNs.

Recently, reconfigurable intelligent surface (RIS) [26]-[28], as a passive and low-energy-consumption technology, has been attracted a lot of attention in wireless communication systems. Specifically, an RIS integrates many passive reflecting elements that can independently change the propagation direction of the electromagnetic waves without signal processing [29]-[31]. Moreover, information exchange can be achieved between a smart controller of RIS and the BS [32], [33]. The signals from the BS to the RIS can be reflected to the destination by reconfiguring the surrounding radio environment. In other words, the RIS can maximize the signal strength of receivers by properly adjusting the reflecting elements of RIS (e.g., phase shifts). Since the RIS has the advantage of altering the radio propagation environment for enhancing the end-toend signal strength in a passive and low-energy-consumption manner, this makes RIS become a promising solution to improve the communication performance of WPCNs.

There are some works on RIS-assisted WPCNs [34]-[37]. For example, the authors in [34] studied a sum-rate maximization RA problem by jointly optimizing the time scheduling and the phase-shift matrix of the RIS. But the fairness of WDs was not considered in [34]. Considering a more practical case, the authors in [35] and [36] investigated the fairnessbased RA problem by maximizing the minimum throughput and the weighted sum rate, respectively. However, the above works [35] and [36] ignored the energy consumption of RIS. Considering the energy consumption of circuit operation, the authors in [37] studied a sum-rate maximization problem by jointly optimizing the phase shifts of the RIS and network resource in a self-sustainable RIS-assisted WPCN, where the RIS can simultaneously harvest the energy from the HAP and reflect the wireless signals to the receivers. However, most of the existing works [34]-[37] employed the HAP to transfer wireless energy and receive wireless information so that it leads to a 'doubly near-far' problem ${ }^{1}$. Moreover, most of the existing works aim to improve the system rate while neglecting system $\mathrm{EE}$, which is also a critical issue for $\mathrm{EH}$ based communication systems ${ }^{2}$.

In this paper, we study the EE-based maximization RA problem for an RIS-assisted WPCN by jointly optimizing radio resource and passive beamforming, where the dedicated power station (PS) and the information receiver (IR) are separated ${ }^{3}$. The main contributions are summarized as follows:

- We design a separated energy/information transceiver for a multiuser RIS-assisted WPCN with a HTT protocol, where multiple WDs first harvest energy from a multiantenna dedicated PS through the RIS and then transmit their wireless signals to the IR by using the harvested energy. Considering the minimum throughput and the $\mathrm{EH}$ constraint of each WD, the maximum transmit power of the PS, as well as the impact of circuit power consumption of the RIS, the total EE maximization problem of WDs is formulated. The formulated problem becomes a fractional optimization problem with coupled variables, which is challenging to solve ${ }^{4}$.

- To tackle this challenging problem, Dinkelbach's method is firstly used to transform the fractional problem into an equivalent subtractive form. Then, an alternating optimization approach is used to decompose the EE maximization problem into two subproblems, i.e., the radio RA subproblem with the fixed passive beamforming and the passive beamforming subproblem with the fixed radio RA parameters. Based on effective variable substitutions, the radio RA subproblem is transformed into a convex one, where the closed-form solutions are obtained by using Lagrange dual method. Furthermore, the passive beamforming subproblem is transformed into a standard convex semidefinite programming (SDP) problem by employing effective variable substitutions and the semidefinite relaxation (SDR) method. Finally, a Dinkelbach-based iterative algorithm and the computa-

${ }^{1}$ In the RIS-assisted WPCN with a HAP, the harvested energy of the fardistance WD under the EH phase is smaller than that of the near-distance WD. However, under the data transmission phase, the transmit power from the fardistance WD to the HAP is much higher than that from the near-distance WD to the HAP. This phenomenon may cause a certain unfairness.

${ }^{2}$ For an EH-based communication network, the amount of the harvested energy and the energy-conversion efficiency are extremely low, so that considering a trade-off between data rates and energy consumption becomes an important design aspect for overall performance improvement.

${ }^{3}$ The separated PS and the IR can well overcome the 'doubly near-far' phenomenon. The reason is that under the information transmission phase, the WD with less harvested energy may be near to the IR. The transmission quality can be guaranteed. In other words, a WD far from a PS may require less energy to achieve similar communication quality as the WD with more harvested energy.

${ }^{4}$ The problem is challenging to solve: i) The fractional objective function is difficult to deal with; ii) the joint optimization problem of the transmit power, transmission time, the active beamforming, and the passive beamforming is challenging for finding a suitable convex form; iii) the minimum throughput constraint is important to guarantee the quality of service (QoS) of each WD, but it is non-convex due to the coupled transmit power and transmission time, which have been separately considered. 


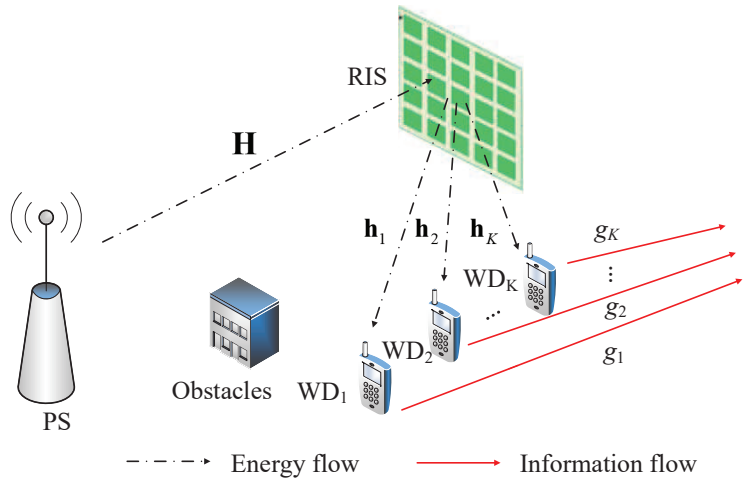

Fig. 1. A downlink multiuser RIS-assisted WPCN.

tional complexity of the proposed algorithm are provided.

- Simulation results demonstrate that the proposed algorithm has fast convergence and higher EE compared with the baseline algorithm and the existing algorithm.

The rest of this paper is structured as follows. The system model and problem formulation are presented in Section II. In Section III, we design an EE-based maximization RA algorithm. Section IV gives simulation results. The paper is concluded in Section V.

\section{System Model And Problem Formulation}

We consider a multiuser RIS-assisted WPCN, as shown in Fig 1, which consists of one PS with $M$ antennas, one RIS, $K$ single-antenna WDs with the EH function and one IR with a single-antenna. The direct link from the PS to the WDs is blocked by obstacles, such as high buildings [38], [39]. The transmission frame is given in Fig. 2, where the time frame $T$ is divided into wireless energy transfer (WET) time (i.e., phase I) and wireless information transmission (WIT) time (i.e., phase II). During phase I, the PS transmits wireless energy to the RIS with $N$ passive reflecting elements. Then, the RIS can reflect energy signals to multiple WDs in the downlink. During phase II, WDs use the harvested energy to transmit wireless information to the IR via a TDMA manner. Defining the set of WDs as $\mathcal{K}=\{1,2, \cdots, K\}$, and the set of reflecting elements as $\mathcal{N}=\{1,2, \cdots, N\}$. All channels are assumed to follow a quasi-static flat fading model, where all channel coefficients keep constant during each block $T$ but vary from one block to another block. All channels are independent of each other. Additionally, vectors and matrices are denoted by bold lowercase letters and bold uppercase letters, respectively. $\|\cdot\|$ denotes the Euclidean norm of a complex vector. $\operatorname{Tr}(\mathbf{X})$ denotes the trace of $\mathbf{X}$. $\mathbf{X}^{\mathrm{T}}$ denotes the transpose of $\mathbf{X} . \mathbf{X}^{\mathrm{H}}$ denotes the conjugate transpose of $\mathbf{X} . \mathbf{X} \succeq \mathbf{0}$ means that $\mathbf{X}$ is a positive semi-define matrix. $\operatorname{diag}(\mathbf{X})$ denotes a vector whose elements are extracted from the diagonal elements of $\mathbf{X}$.

According to the frame structure, the total time satisfies

$$
t_{0}+\sum_{k=1}^{K} t_{k} \leq T .
$$

where $t_{0}$ denotes the WET time, $t_{k}$ denotes the WIT time of WD $k$.

\begin{tabular}{|c|c|c|c|c|} 
& \multicolumn{5}{|c|}{ Phase II } \\
\hline $\begin{array}{l}\mathrm{PS} \longrightarrow \mathrm{RIS} \\
\mathrm{RIS} \longrightarrow \mathrm{WDs}\end{array}$ & $\mathrm{WD}_{1} \longrightarrow \mathrm{IR}$ & $\mathrm{WD}_{2} \longrightarrow \mathrm{IR}$ & $\cdots$ & $\mathrm{WD}_{K} \longrightarrow \mathrm{IR}$ \\
\hline$t_{0}$ & $t_{1}$ & $t_{2}$ & $\ldots$ & $t_{K}$ \\
\hline & \multicolumn{5}{|c}{$T-$ WIT } \\
\hline
\end{tabular}

Fig. 2. The transmission frame.

\section{A. Phase I: WET}

During phase I, the PS sends the energy signals to the RIS meanwhile the RIS reflects the obtained signals to the EH WDs. The harvested energy of WDs is used to support the circuit operation and data transmission for phase II. Defining $\mathbf{s} \in \mathbb{C}^{M \times 1}$ as the pseudo-random energy signal transmitted by the PS, the maximum transmit power is limited by

$$
\operatorname{Tr}\left(\mathbb{E}\left[\mathbf{s s}^{\mathrm{H}}\right]\right)=\operatorname{Tr}(\mathbf{W}) \leq P^{\max },
$$

where $\mathbf{W} \in \mathbb{C}^{M \times M}$ denotes the energy beamforming matrix, and $\mathbf{W} \succeq 0$. $P^{\max }$ denotes the maximum transmit power threshold of the PS.

Denoting $\boldsymbol{v}=\left[v_{1}, v_{2}, \cdots, v_{N}\right]^{\mathrm{H}}$ as the reflection coefficients of the RIS, where $v_{n}=e^{j \theta_{n}}$ and $\left|v_{n}\right|=1 . \theta_{n} \in[0,2 \pi)$ denotes the phase shift of the $n$-th reflecting element [29]. We define $\Theta=\operatorname{diag}(\boldsymbol{v})$ as the reflection matrix of the RIS and can obtain the received energy signal of WD $k$, i.e.,

$$
y_{k}^{\mathrm{EH}}=\mathbf{h}_{k}^{\mathrm{H}} \mathbf{\Theta H} \mathbf{s}+n_{k},
$$

where $\mathbf{H} \in \mathbb{C}^{N \times M}$ and $\mathbf{h}_{k} \in \mathbb{C}^{N \times 1}$ represent the channel matrix and the channel vector from the PS to the RIS and from the RIS to the $k$-th WD, respectively. $n_{k}$ denotes the additive white Gaussian noise (AWGN) of WD $k$ with zero mean and variance $\delta_{k}^{2}$, namely, $n_{k} \sim \mathcal{C N}\left(0, \delta_{k}^{2}\right)$.

Since the background noise is always small, $n_{k}$ can be ignored [37]. Thus, the harvested energy at the $k$-th WD is

$$
E_{k}^{\mathrm{EH}}=\chi t_{0} \mathbb{E}\left[\left|y_{k}^{\mathrm{EH}}\right|^{2}\right]=\chi t_{0}\left\{\left(\mathbf{h}_{k}^{\mathrm{H}} \mathbf{\Theta H}\right) \mathbf{W}\left(\mathbf{h}_{k}^{\mathrm{H}} \mathbf{\Theta H}\right)^{\mathrm{H}}\right\},
$$

where $\chi \in[0,1]$ denotes the energy conversion efficiency of each $\mathrm{WD}^{5}$.

Defining $P^{\mathrm{PS}}$ and $P_{e}$ as the circuit power consumption of the PS and each reflecting element, respectively. Thus, the total

5 The linear EH model is always used in EH-based wireless networks [31], [35]-[37]. Moreover, we focus on the investigation of the EE maximization problem in RIS-assisted WPCNs and exploring how to jointly optimize radio resource and the passive beamforming of the RIS to maximize the total EE of WDs. The formulated problem is a fractional optimization problem with coupled variables, which is challenging to solve. If we apply the nonlinear EH model, which makes the optimization problem more complex and hard to solve. Besides, the linear EH model can be considered as a special case of the nonlinear EH model with a piece-wise linear form [40] in some degree. Moreover, according to [41], the linear EH model is accurate for the practical $\mathrm{EH}$ circuits when the input power is small. For the above reasons, the linear $\mathrm{EH}$ model is adopted in this paper. 
energy consumption under phase I can be formulated as

$$
\begin{aligned}
Q^{\mathrm{Phase}-\mathrm{I}}\left(\mathbf{W}, t_{0}, \boldsymbol{\Theta}\right) & =t_{0}\left(P^{\mathrm{PS}}+N P_{e}\right)+\sum_{k=1}^{K} t_{0} P_{k}^{\mathrm{C}} \\
& +t_{0} \operatorname{Tr}(\mathbf{W})-\sum_{k=1}^{K} E_{k}^{\mathrm{EH}}
\end{aligned}
$$

where $P_{k}^{\mathrm{C}}$ denotes the circuit power consumption of WD $k$.

\section{B. Phase II: WIT}

During phase II, each WD transmits its own information to the IR in a TDMA manner. Defining $s_{k}^{\mathrm{WD}}$ as the transmission symbol of WD $k$, satisfies $\mathbb{E}\left[\left|s_{k}^{\mathrm{WD}}\right|^{2}\right]=1$, the transmission signal of WD $k$ is given by

$$
x_{k}^{\mathrm{WD}}=\sqrt{p_{k}} s_{k}^{\mathrm{WD}},
$$

where $p_{k} \geq 0$ denotes the transmit power of WD $k$. The received signal from WD $k$ to the IR is

$$
y_{k}^{\mathrm{IR}}=g_{k} x_{k}^{\mathrm{WD}}+n^{\mathrm{IR}},
$$

where $g_{k}$ denotes the channel coefficient from WD $k$ to the IR. $n^{\mathrm{IR}}$ denotes the AWGN at the IR with zero mean and variance $\delta^{2}$, namely $n^{\mathrm{IR}} \sim \mathcal{C N}\left(0, \delta^{2}\right)$.

The achievable throughput of WD $k$ is

$$
R_{k}=t_{k} \log _{2}\left(1+\frac{p_{k}\left|g_{k}\right|^{2}}{\delta^{2}}\right)
$$

Denoting $\boldsymbol{t}=\left[t_{1}, t_{2}, \cdots, t_{K}\right]^{\mathrm{T}}$ and $\boldsymbol{p}=\left[p_{1}, p_{2}, \cdots, p_{K}\right]^{\mathrm{T}}$ as the time allocation vector and the transmit power vector of WDs, respectively. Accordingly, the sum throughput of all WDs is

$$
R(\boldsymbol{t}, \boldsymbol{p})=\sum_{k=1}^{K} R_{k}=\sum_{k=1}^{K} t_{k} \log _{2}\left(1+\frac{p_{k}\left|g_{k}\right|^{2}}{\delta^{2}}\right) .
$$

Since the transmit power of WD $k$ (e.g., $p_{k}$ ) is limited by its harvested energy, we have the following transmit power constraint

$$
p_{k} t_{k}+P_{k}^{\mathrm{C}}\left(t_{0}+t_{k}\right) \leq E_{k}^{\mathrm{EH}} .
$$

Defining $P^{\mathrm{IR}}$ as the circuit power consumption of the IR, the total energy consumption under phase II is formulated as

$$
Q^{\mathrm{Phase}-\mathrm{II}}(\boldsymbol{t}, \boldsymbol{p})=\sum_{k=1}^{K} t_{k} p_{k}+\sum_{k=1}^{K} t_{k} P_{k}^{\mathrm{C}}+\sum_{k=1}^{K} t_{k} P^{\mathrm{IR}}
$$

Based on (5) and (11), the total energy consumption of the whole system is given by

$$
\begin{aligned}
& Q^{\text {total }}\left(\mathbf{W}, t_{0}, \boldsymbol{t}, \boldsymbol{p}, \boldsymbol{\Theta}\right)=Q^{\text {Phase-I }}(\cdot)+Q^{\text {Phase-II }}(\cdot) \\
& =t_{0}\left(P^{\mathrm{PS}}+N P_{e}\right)+\sum_{k=1}^{K}\left(t_{0}+t_{k}\right) P_{k}^{\mathrm{C}}+t_{0} \operatorname{Tr}(\mathbf{W})+ \\
& \sum_{k=1}^{K} t_{k}\left(p_{k}+P^{\mathrm{IR}}\right)-\sum_{k=1}^{K} \chi t_{0}\left\{\left(\mathbf{h}_{k}^{\mathrm{H}} \mathbf{\Theta} \mathbf{H}\right) \mathbf{W}\left(\mathbf{h}_{k}^{\mathrm{H}} \mathbf{\Theta} \mathbf{H}\right)^{\mathrm{H}}\right\} .
\end{aligned}
$$

\section{Problem Formulation}

To reflect the impact of energy consumption on overall performance and guarantee the QoS of each WD, an EE-based
RA problem with the minimum throughput constraint can be formulated as

$$
\begin{array}{cl}
\max _{\mathbf{W}, t_{0}, \boldsymbol{t}, \boldsymbol{p}, \boldsymbol{\Theta}} \frac{R(\boldsymbol{t}, \boldsymbol{p})}{Q^{\mathrm{total}}\left(\mathbf{W}, t_{0}, \boldsymbol{t}, \boldsymbol{p}, \boldsymbol{\Theta}\right)} \\
\text { s.t. } & C_{1}: p_{k} t_{k}+P_{k}^{\mathrm{C}}\left(t_{0}+t_{k}\right) \leq E_{k}^{\mathrm{EH}}, \\
C_{2}: t_{0}+\sum_{k=1}^{K} t_{k} \leq T, t_{0} \geq 0, t_{k} \geq 0, \\
C_{3}: t_{k} \log _{2}\left(1+\frac{p_{k}\left|g_{k}\right|^{2}}{\delta^{2}}\right) \geq R_{k}^{\min }, \\
C_{4}: \operatorname{Tr}(\mathbf{W}) \leq P^{\max }, \mathbf{W} \succeq 0, \\
C_{5}:\left|\boldsymbol{\Theta}_{n, n}\right|=1, \forall n,
\end{array}
$$

where $R_{k}^{\min }$ denotes the individual QoS constraint of WD $k$. $C_{1}$ ensures that the energy consumption of WD $k$ does not exceed the harvested energy $E_{k}^{\mathrm{EH}}$. $C_{2}$ denotes the total time constraint. $C_{3}$ is the minimum throughput constraint of each WD. $C_{4}$ limits the maximum transmit power of the PS. $C_{5}$ is the phase-shift constraint of the RIS ${ }^{6}$. Problem (13) is nonconvex due to the fractional objective function and the coupled optimization variables.

\section{ENERGY-EFFICIENT RA ALGORITHM}

To solve problem (13), Dinkelbach's method is employed to transform the objective function into a subtractive form [42]. Accordingly, an alternating optimization approach [30] is used to decompose the problem into two subproblems: i) the radio RA subproblem with the fixed passive beamforming $\Theta$; ii) the passive beamforming optimization subproblem with the fixed parameters $\left(\mathbf{W}, t_{0}, \boldsymbol{t}, \boldsymbol{p}\right)$. These two subproblems are converted into convex problems which are resolved by using the Lagrange dual method and the SDP method, respectively.

\section{A. Transformation of the Objective Function}

It is observed that the objective function in problem (13) is a fractional form that is hard to solve directly. Based on Dinkelbach's method [42], [43], we define $\eta$ as the system EE, namely

$$
\eta=\frac{R(\boldsymbol{t}, \boldsymbol{p})}{Q^{\mathrm{total}}\left(\mathbf{W}, t_{0}, \boldsymbol{t}, \boldsymbol{p}, \boldsymbol{\Theta}\right)} .
$$

Defining $\eta^{*}$ as the optimal EE, $\eta^{*}$ can be achieved if and only if it satisfies

$$
\max _{\mathbf{W}, t_{0}, \boldsymbol{t}, \boldsymbol{p}, \Theta} R(\boldsymbol{t}, \boldsymbol{p})-\eta^{*} Q^{\text {total }}\left(\mathbf{W}, t_{0}, \boldsymbol{t}, \boldsymbol{p}, \boldsymbol{\Theta}\right)=0 .
$$

As a result, for a given $\eta$, problem (13) can be solved by the following parameters optimization problem

$$
\begin{array}{ll} 
& \max _{\mathbf{W}, t_{0}, \boldsymbol{t}, \boldsymbol{p}, \boldsymbol{\Theta}} R(\boldsymbol{t}, \boldsymbol{p})-\eta Q^{\text {total }}\left(\mathbf{W}, t_{0}, \boldsymbol{t}, \boldsymbol{p}, \boldsymbol{\Theta}\right) \\
\text { s.t. } C_{1}-C_{5} .
\end{array}
$$

6 In fact, the reflection coefficients of the RIS include both amplitudes and phase shifts, i.e., $\beta_{n} e^{j \theta_{n}}$, where $\beta_{n} \in[0,1]$ and $\theta_{n} \in[0,2 \pi)$ denote the reflection amplitude and phase shift of the $n$-th element, respectively. Similar to the existing works [34]-[37], we set $\beta_{n}=1$, and the phase shifts satisfy $\left|\Theta_{n, n}\right|=1$. As a result, each reflecting element of the RIS can provide the phase-shift optimization for changing radio channel environments. 
From (14) to (16), we give the transformation process of the objective function in problem (13) from the fractional form to an equivalent subtractive form. To better illustrate the updating process of $\eta$, we provide a flow chart as shown in Fig. 3, where $\eta^{(0)}=0$ denotes the initial value of $\eta, \eta^{(i)}$ denotes the $i$-th iteration value of $\eta, \varepsilon$ denotes the convergence precision. Problem (16) is still a non-convex problem due to the coupled variables (e.g., $\left(\mathbf{W}, t_{0}, \boldsymbol{\Theta}\right)$ is coupled, $(\boldsymbol{t}, \boldsymbol{p})$ is coupled). Then, we use the alternating optimization approach [30] to solve problem (16).

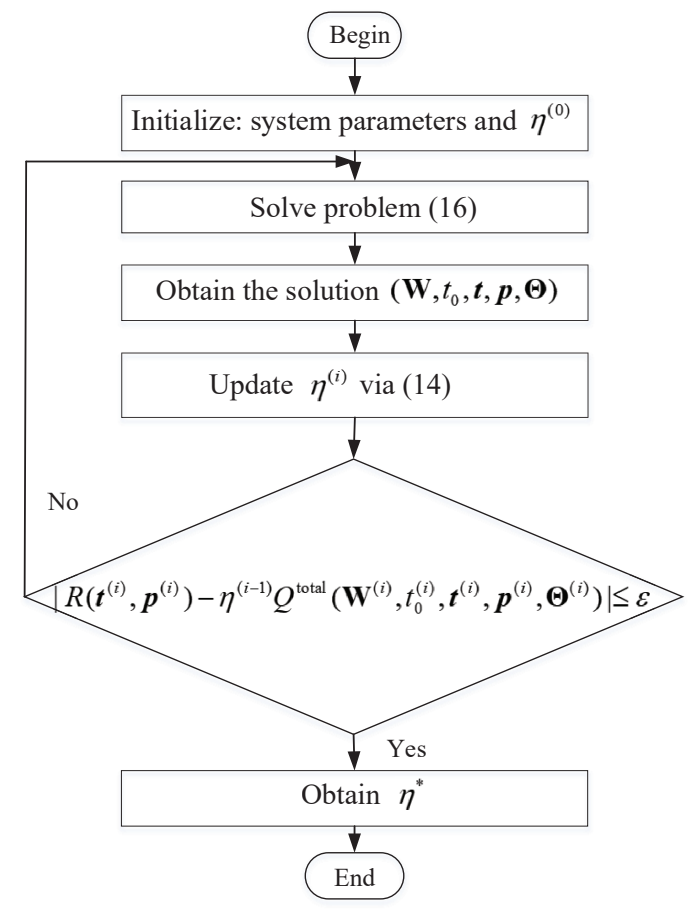

Fig. 3. The flow chart of updating $\eta$.

\section{B. Radio RA Subproblem}

Under the fixed $\Theta$, the cascaded channel from the PS to the $k$-th WD can be defined as $\mathbf{G}_{k}=\mathbf{h}_{k}^{\mathrm{H}} \boldsymbol{\Theta H}$. Substituting (4) into problem (16), we can obtain the following optimization problem

$$
\begin{aligned}
& \max _{\mathbf{W}, t_{0}, \boldsymbol{t}, \boldsymbol{p}} R(\boldsymbol{t}, \boldsymbol{p})-\eta Q^{\text {total }}\left(\mathbf{W}, t_{0}, \boldsymbol{t}, \boldsymbol{p}\right) \\
& \text { s.t. } \\
& C_{2}-C_{4}, \\
& \tilde{C}_{1}: p_{k} t_{k}+P_{k}^{\mathrm{C}}\left(t_{0}+t_{k}\right) \leq \chi t_{0}\left(\mathbf{G}_{k} \mathbf{W G}_{k}^{\mathrm{H}}\right) .
\end{aligned}
$$

Problem (17) is still a non-convex optimization problem due to the coupled variables (i.e., $\mathbf{W}, t_{0}, t_{k}, p_{k}$ ) in the objective function and the constraints. To resolve the problem, define the auxiliary variables $\overline{\mathbf{W}}=t_{0} \mathbf{W}, f_{k}(\overline{\mathbf{W}})=\chi\left(\mathbf{G}_{k} \overline{\mathbf{W}}_{k}^{\mathrm{H}}\right)$ and $\overline{\boldsymbol{p}}=\left[\bar{p}_{1}, \bar{p}_{2}, \cdots, \bar{p}_{K}\right]^{\mathrm{T}}$ (i.e., $\bar{p}_{k}=p_{k} t_{k}$ ), based on (9) and
(12), problem (17) can be written as

$$
\begin{gathered}
\max _{\overline{\mathbf{W}}, t_{0}, \boldsymbol{t}, \overline{\boldsymbol{p}}} \sum_{k=1}^{K} t_{k} \log _{2}\left(1+\frac{\bar{p}_{k}\left|g_{k}\right|^{2}}{t_{k} \delta^{2}}\right)-\eta\left\{\sum_{k=1}^{K} \bar{p}_{k}\right. \\
+t_{0}\left(P^{\mathrm{PS}}+N P_{e}\right)+\sum_{k=1}^{K}\left(t_{0}+t_{k}\right) P_{k}^{\mathrm{C}} \\
\left.+\sum_{k=1}^{K} t_{k} P^{\mathrm{IR}}+\operatorname{Tr}(\overline{\mathbf{W}})-\sum_{k=1}^{K} f_{k}(\overline{\mathbf{W}})\right\} \\
\text { s.t. } C_{2}, \bar{C}_{1}: \bar{p}_{k}+P_{k}^{\mathrm{C}}\left(t_{0}+t_{k}\right) \leq f_{k}(\overline{\mathbf{W}}), \\
\bar{C}_{3}: t_{k} \log _{2}\left(1+\frac{\bar{p}_{k}\left|g_{k}\right|^{2}}{t_{k} \delta^{2}}\right) \geq R_{k}^{\min }, \\
\bar{C}_{4}: \operatorname{Tr}(\overline{\mathbf{W}}) \leq t_{0} P^{\max } .
\end{gathered}
$$

Problem (18) is a standard convex optimization problem that can be solved by using the Lagrangian dual method [44]. The Lagrangian function of problem (18) is given by

$$
\begin{aligned}
& L\left(\overline{\mathbf{W}}, t_{0}, \boldsymbol{t}, \overline{\boldsymbol{p}}, \boldsymbol{\varphi}, \boldsymbol{\beta}, \kappa, \mu\right)=\sum_{k=1}^{K} t_{k} \log _{2}\left(1+\frac{\bar{p}_{k}\left|g_{k}\right|^{2}}{t_{k} \delta^{2}}\right) \\
& -\eta\left\{t_{0}\left(P^{\mathrm{PS}}+N P_{e}\right)+\sum_{k=1}^{K}\left(t_{0}+t_{k}\right) P_{k}^{\mathrm{C}}+\sum_{k=1}^{K} \bar{p}_{k}\right. \\
& \left.+\sum_{k=1}^{K} t_{k} P^{\mathrm{IR}}+\operatorname{Tr}(\overline{\mathbf{W}})-\sum_{k=1}^{K} f_{k}(\overline{\mathbf{W}})\right\} \\
& +\sum_{k=1}^{K} \varphi_{k}\left\{f_{k}(\overline{\mathbf{W}})-\left(\bar{p}_{k}+P_{k}^{\mathrm{C}}\left(t_{0}+t_{k}\right)\right)\right\} \\
& +\sum_{k=1}^{K} \beta_{k}\left\{t_{k} \log _{2}\left(1+\frac{\bar{p}_{k}\left|g_{k}\right|^{2}}{t_{k} \delta^{2}}\right)-R_{k}^{\min }\right\} \\
& +\kappa\left(T-t_{0}-\sum_{k=1}^{K} t_{k}\right)+\mu\left\{t_{0} P^{\max }-\operatorname{Tr}(\overline{\mathbf{W}})\right\}
\end{aligned}
$$

where $\boldsymbol{\varphi}=\left[\varphi_{1}, \varphi_{2}, \cdots, \varphi_{K}\right]^{\mathrm{T}}, \boldsymbol{\beta}=\left[\beta_{1}, \beta_{2}, \cdots, \beta_{K}\right]^{\mathrm{T}}, \kappa$, and $\mu$ are non-negative Lagrange multipliers. Therefore, the dual function is

$$
\Gamma(\boldsymbol{\varphi}, \boldsymbol{\beta}, \kappa, \mu)=\max _{\overline{\mathbf{W}}, t_{0}, \boldsymbol{t}, \overline{\boldsymbol{p}}} L\left(\overline{\mathbf{W}}, t_{0}, \boldsymbol{t}, \overline{\boldsymbol{p}}, \boldsymbol{\varphi}, \boldsymbol{\beta}, \kappa, \mu\right) .
$$

The dual problem is given as

$$
\begin{aligned}
& \min _{\boldsymbol{\varphi}, \boldsymbol{\beta}, \kappa, \mu} \Gamma(\boldsymbol{\varphi}, \boldsymbol{\beta}, \kappa, \mu) \\
& \text { s.t. } \boldsymbol{\varphi} \succeq \mathbf{0}, \boldsymbol{\beta} \succeq \mathbf{0}, \kappa \geq 0, \mu \geq 0 .
\end{aligned}
$$

To obtain the optimal $\overline{\mathbf{W}}^{*}$, (19) can be rewritten as

$$
\begin{aligned}
L\left(\overline{\mathbf{W}}, t_{0}, \boldsymbol{t}, \overline{\boldsymbol{p}}, \boldsymbol{\varphi}, \boldsymbol{\beta}, \kappa, \mu\right) & =\bar{L}\left(t_{0}, \boldsymbol{t}, \overline{\boldsymbol{p}}, \boldsymbol{\varphi}, \boldsymbol{\beta}, \kappa, \mu\right) \\
& +\operatorname{Tr}(\overline{\mathbf{G}} \overline{\mathbf{W}}),
\end{aligned}
$$

where $\overline{\mathbf{G}}=\sum_{k=1}^{K}\left(\eta+\varphi_{k}\right) \chi \mathbf{G}_{k}^{\mathrm{H}} \mathbf{G}_{k}-(\eta+\mu) \mathbf{I}_{M}$, and

$$
\begin{aligned}
& \bar{L}\left(t_{0}, \boldsymbol{t}, \overline{\boldsymbol{p}}, \boldsymbol{\varphi}, \boldsymbol{\beta}, \kappa, \mu\right)=\sum_{k=1}^{K}\left(1+\beta_{k}\right) t_{k} \log _{2}\left(1+\frac{\bar{p}_{k}\left|g_{k}\right|^{2}}{t_{k} \delta^{2}}\right) \\
& +\kappa\left(T-t_{0}-\sum_{k=1}^{K} t_{k}\right)-\eta\left\{t_{0}\left(P^{\mathrm{PS}}+N P_{\mathrm{e}}\right)+\sum_{k=1}^{K} \bar{p}_{k}\right. \\
& \left.+\sum_{k=1}^{K}\left(t_{0}+t_{k}\right) P_{k}^{\mathrm{C}}+\sum_{k=1}^{K} t_{k} P^{\mathrm{IR}}\right\}+\mu t_{0} P^{\max } \\
& -\sum_{k=1}^{K} \varphi_{k}\left\{\bar{p}_{k}+P_{k}^{\mathrm{C}}\left(t_{0}+t_{k}\right)\right\}-\sum_{k=1}^{K} \beta_{k} R_{k}^{\min } .
\end{aligned}
$$


As a result, the solution of $\overline{\mathbf{W}}$ can be obtained by using the following lemma.

Lemma 1: Defining $\mathbf{G}=\sum_{k=1}^{K}\left(\eta+\varphi_{k}\right) \chi \mathbf{G}_{k}^{\mathrm{H}} \mathbf{G}_{k}$, for the fixed $\left(t_{0}, \eta, \varphi_{k}, \mu\right), \overline{\mathbf{W}}^{*}$ and $\mu^{*}$ are

$$
\left\{\begin{array}{l}
\overline{\mathbf{W}}^{*}=t_{0} P^{\max } \mathbf{u}_{1} \mathbf{u}_{1}{ }^{\mathrm{H}}, \\
\mu^{*}=\sigma_{1}-\eta
\end{array}\right.
$$

where $\sigma_{1}$ and $\mathbf{u}_{1}$ are the largest eigenvalue and its associated eigenvector of $\mathbf{G}$, respectively. Since $\overline{\mathbf{W}}=t_{0} \mathbf{W}$, the closedform solution of $\mathbf{W}^{*}$ is

$$
\mathbf{W}^{*}=P^{\max } \mathbf{u}_{1} \mathbf{u}_{1}^{\mathrm{H}} .
$$

Proof: See Appendix A.

Furthermore, in order to get the updating expression of $t_{0}$, we take the partial derivative of $L\left(\overline{\mathbf{W}}, t_{0}, \boldsymbol{t}, \overline{\boldsymbol{p}}, \boldsymbol{\varphi}, \boldsymbol{\beta}, \kappa, \mu\right)$ with respect to $t_{0}$, namely

$$
\begin{aligned}
\frac{\partial L\left(\overline{\mathbf{W}}, t_{0}, \boldsymbol{t}, \overline{\boldsymbol{p}}, \boldsymbol{\varphi}, \boldsymbol{\beta}, \kappa, \mu\right)}{\partial t_{0}} & =\mu P^{\max }-\kappa-\sum_{k=1}^{K} \varphi_{k} P_{k}^{\mathrm{C}} \\
& -\eta\left\{\left(P^{\mathrm{PS}}+N P_{e}\right)-\sum_{k=1}^{K} P_{k}^{\mathrm{C}}\right\} .
\end{aligned}
$$

Since (25) is a linear function of $t_{0}, t_{0}$ can be updated by using the subgradient method [45], namely

$$
t_{0}^{(l+1)}=\left[t_{0}^{(l)}-\Delta_{1}^{(l)} \times \frac{\partial L\left(\overline{\mathbf{W}}, t_{0}, \boldsymbol{t}, \overline{\boldsymbol{p}}, \boldsymbol{\varphi}, \boldsymbol{\beta}, \kappa, \mu\right)}{\partial t_{0}}\right]^{+},
$$

where $[x]^{+} \triangleq \max (0, x) . l$ is the iteration number. $\Delta_{1}$ represents the step size.

To obtain the optimal $p_{k}^{*}$ and $t_{k}^{*}$, (19) can be rewritten as

$$
\begin{aligned}
& L\left(\overline{\mathbf{W}}, t_{0}, \boldsymbol{t}, \overline{\boldsymbol{p}}, \boldsymbol{\varphi}, \boldsymbol{\beta}, \kappa, \mu\right)=\sum_{k=1}^{K} L_{k}\left(t_{k}, \bar{p}_{k}, \varphi_{k}, \beta_{k}, \kappa\right) \\
& -\eta\left\{t_{0}\left(P^{\mathrm{PS}}+N P_{e}\right)+\operatorname{Tr}(\overline{\mathbf{W}})-\sum_{k=1}^{K} f_{k}(\overline{\mathbf{W}})\right. \\
& \left.+\sum_{k=1}^{K} t_{0} P_{k}^{\mathrm{C}}\right\}+\kappa\left(T-t_{0}\right)+\mu\left\{t_{0} P^{\mathrm{max}}-\operatorname{Tr}(\overline{\mathbf{W}})\right\} \\
& +\sum_{k=1}^{K} \varphi_{k}\left\{f_{k}(\overline{\mathbf{W}})-P_{k}^{\mathrm{C}} t_{0}\right\}-\sum_{k=1}^{K} \beta_{k} R_{k}^{\min },
\end{aligned}
$$

where

$$
\begin{aligned}
& L_{k}\left(t_{k}, \bar{p}_{k}, \varphi_{k}, \beta_{k}, \kappa\right)=t_{k}\left(1+\beta_{k}\right) \log _{2}\left(1+\frac{\bar{p}_{k}\left|g_{k}\right|^{2}}{t_{k} \delta^{2}}\right) \\
& -t_{k}\left\{\kappa+\eta P^{\mathrm{IR}}+P_{k}^{\mathrm{C}}\left(\eta+\varphi_{k}\right)\right\}-\bar{p}_{k}\left(\eta+\varphi_{k}\right) .
\end{aligned}
$$

By taking the partial derivative of $L_{k}\left(t_{k}, \bar{p}_{k}, \varphi_{k}, \beta_{k}, \kappa\right)$ with respect to $\bar{p}_{k}$ and $t_{k}$, respectively, we have

$$
\begin{aligned}
& \frac{\partial L_{k}\left(t_{k}, \bar{p}_{k}, \varphi_{k}, \beta_{k}, \kappa\right)}{\partial \bar{p}_{k}}=\frac{1+\beta_{k}}{\ln 2}\left(\frac{t_{k}\left|g_{k}\right|^{2}}{t_{k} \delta^{2}+\bar{p}_{k}\left|g_{k}\right|^{2}}\right) \\
&-\eta-\varphi_{k} . \\
& \frac{\partial L_{k}\left(t_{k}, \bar{p}_{k}, \varphi_{k}, \beta_{k}, \kappa\right)}{\partial t_{k}}=\left(1+\beta_{k}\right) \log _{2}\left(1+\frac{p_{k}\left|g_{k}\right|^{2}}{\delta^{2}}\right) \\
&-\left\{\kappa+\eta P^{\mathrm{IR}}+P_{k}^{\mathrm{C}}\left(\eta+\varphi_{k}\right)\right\}-p_{k}\left(\eta+\varphi_{k}\right) .
\end{aligned}
$$

Based on Karush-Kuhn-Tucker (KKT) conditions [45], the closed-form solution of $p_{k}$ can be obtained by $\frac{\partial L_{k}\left(t_{k}, \bar{p}_{k}, \varphi_{k}, \beta_{k}, \kappa\right)}{\partial \bar{p}_{k}}=0$, i.e.,

$$
p_{k}^{*}=\frac{\bar{p}_{k}^{*}}{t_{k}}=\left[\frac{1+\beta_{k}}{\ln 2\left(\eta+\varphi_{k}\right)}-\frac{\delta^{2}}{\left|g_{k}\right|^{2}}\right]^{+} .
$$

Since (29) is a linear function of $t_{k}$, we have

$$
t_{k}^{(l+1)}=\left[t_{k}^{(l)}-\Delta_{2}^{(l)} \times \frac{\partial L_{k}\left(t_{k}, \bar{p}_{k}, \varphi_{k}, \beta_{k}, \kappa\right)}{\partial t_{k}}\right]^{+},
$$

where $\Delta_{2}$ is the step size. Similarly, Lagrangian multipliers can be updated by

$\varphi_{k}^{(l+1)}=\left[\varphi_{k}^{(l)}-\Delta_{3}^{(l)} \times\left\{f_{k}(\overline{\mathbf{W}})-\left(t_{k} p_{k}+P_{k}^{\mathrm{C}}\left(t_{0}+t_{k}\right)\right)\right\}\right]^{+}$,

$\beta_{k}^{(l+1)}=\left[\beta_{k}^{(l)}-\Delta_{4}^{(l)} \times\left\{t_{k} \log _{2}\left(1+\frac{p_{k}\left|g_{k}\right|^{2}}{\delta^{2}}\right)-R_{k}^{\min }\right\}\right]_{(33)}^{+}$

$$
\kappa^{(l+1)}=\left[\kappa^{(l)}-\Delta_{5}^{(l)} \times\left(T-t_{0}-\sum_{k=1}^{K} t_{k}\right)\right]^{+},
$$

where $\Delta_{3}, \Delta_{4}$, and $\Delta_{5}$ are the corresponding step sizes of Lagrange multipliers.

\section{Passive Beamforming Subproblem}

Defining $\quad \boldsymbol{\Phi}_{k}=\operatorname{diag}\left(\mathbf{h}_{k}^{\mathrm{H}}\right) \mathbf{H}, \quad$ we have $\mathbf{h}_{k}^{\mathrm{H}} \boldsymbol{\Theta H}=\boldsymbol{v}^{\mathrm{H}} \operatorname{diag}\left(\mathbf{h}_{k}^{\mathrm{H}}\right) \mathbf{H}=\boldsymbol{v}^{\mathrm{H}} \boldsymbol{\Phi}_{k}$. Unedr the fixed $\left(\mathbf{W}, t_{0}, \boldsymbol{t}, \boldsymbol{p}\right)$, based on (4) and (13), the passive beamforming subproblem of problem (16) becomes

$$
\begin{aligned}
& \max _{\boldsymbol{v}} \sum_{k=1}^{K} \chi t_{0}\left(\boldsymbol{v}^{\mathrm{H}} \boldsymbol{\Phi}_{k} \mathbf{W} \boldsymbol{\Phi}_{k}^{\mathrm{H}} \boldsymbol{v}\right) \\
\text { s.t. } & \hat{C}_{1}: p_{k} t_{k}+P_{k}^{\mathrm{C}}\left(t_{0}+t_{k}\right) \leq \chi t_{0}\left(\mathbf{v}^{\mathrm{H}} \boldsymbol{\Phi}_{k} \mathbf{W} \boldsymbol{\Phi}_{k}^{\mathrm{H}} \mathbf{v}\right), \\
& \hat{C}_{5}:\left|v_{n}\right|^{2}=1, \forall n .
\end{aligned}
$$

Problem (35) is a non-convex problem, since $\hat{C}_{5}$ is a non-convex quadratic equality constraint. We have $\boldsymbol{v}^{\mathrm{H}} \boldsymbol{\Phi}_{k} \mathbf{W} \boldsymbol{\Phi}_{k}^{\mathrm{H}} \boldsymbol{v}=\operatorname{Tr}\left(\boldsymbol{\Phi}_{k} \mathbf{W} \boldsymbol{\Phi}_{k}^{\mathrm{H}} \boldsymbol{v} \boldsymbol{v}^{\mathrm{H}}\right)$ and define $\mathbf{V}=\boldsymbol{v} \boldsymbol{v}^{\mathrm{H}}$, where $\operatorname{Rank}(\mathbf{V})=1$ and $\mathbf{V} \succeq \mathbf{0}$ [42]. However, the rank-one constraint is non-convex. Based on the SDR method, problem (35) can be relaxed as

$$
\begin{aligned}
& \max _{\mathbf{V}} \sum_{k=1}^{K} \chi t_{0} \operatorname{Tr}\left(\boldsymbol{\Phi}_{k} \mathbf{W} \boldsymbol{\Phi}_{k}^{\mathrm{H}} \mathbf{V}\right) \\
\text { s.t. } & \breve{C}_{1}: p_{k} t_{k}+P_{k}^{\mathrm{C}}\left(t_{0}+t_{k}\right) \leq \chi t_{0} \operatorname{Tr}\left(\boldsymbol{\Phi}_{k} \mathbf{W} \boldsymbol{\Phi}_{k}^{\mathrm{H}} \mathbf{V}\right), \\
& \breve{C}_{5}: \mathbf{V}_{n, n}=1, \forall n, \mathbf{V} \succeq \mathbf{0} .
\end{aligned}
$$

Problem (36) is a standard SDP that can be solved by using convex optimization tools [45]. However, the optimal solution of (36) may not satisfy the rank-one condition, namely $\operatorname{Rank}(\mathbf{V}) \neq 1$. Therefore, the Gaussian randomization method [30], [46] is further employed to solve it. An iterationbased RA algorithm is summarized in Algorithm 1.

Defining $\overline{\mathbf{V}}$ as the optimal solution of problem (36), we can obtain the singular value decomposition $\overline{\mathbf{V}}=\Pi \mathbf{\Pi} \Pi^{\mathrm{H}}$, 


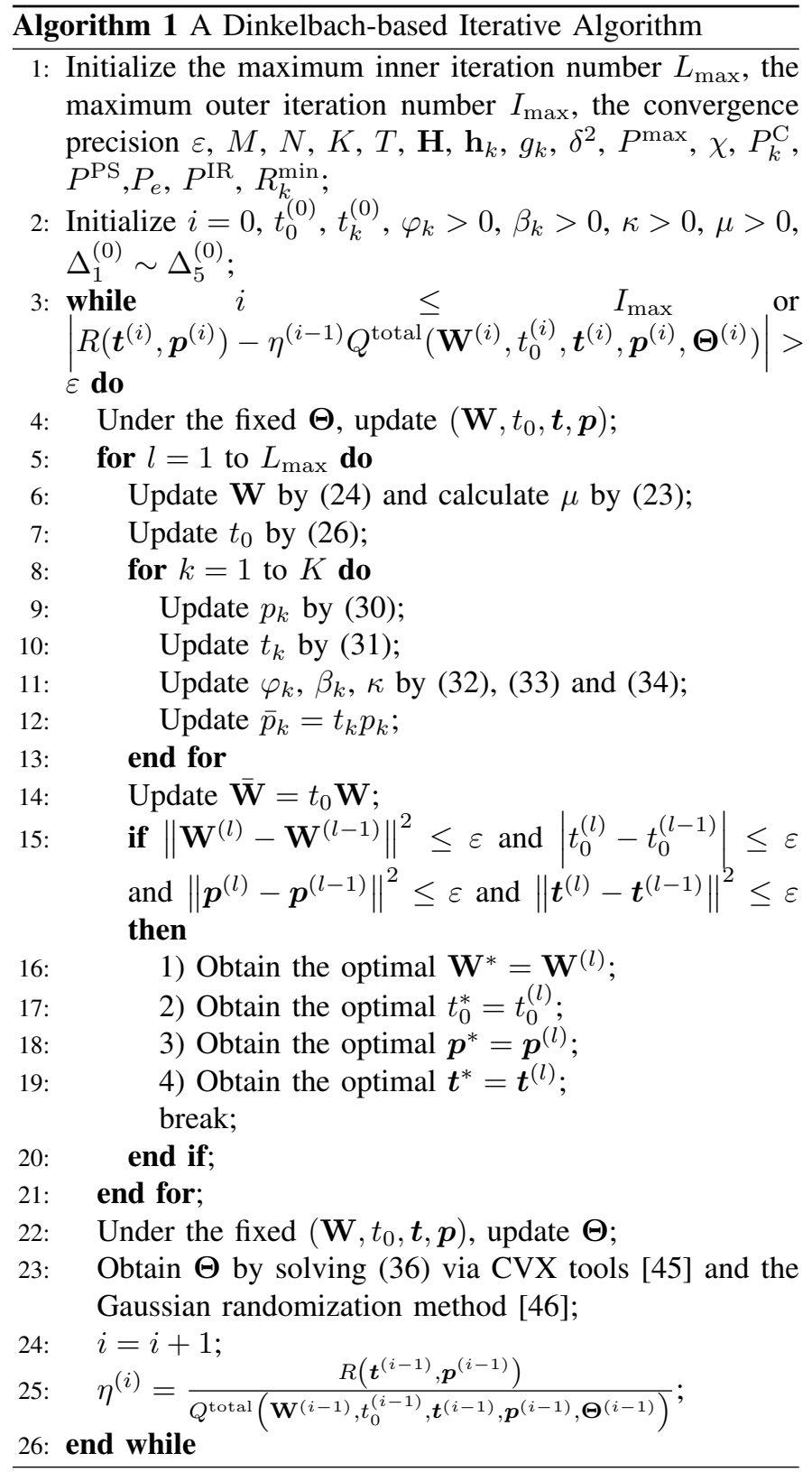

where $\boldsymbol{\Pi}=\left[\boldsymbol{\pi}_{1}, \cdots, \boldsymbol{\pi}_{N}\right]^{\mathrm{H}}$ and $\boldsymbol{\Lambda}=\operatorname{diag}\left(\lambda_{1}, \cdots, \lambda_{N}\right)$ are the unitary matrix and the diagonal matrix, respectively. Then, the suboptimal solution of problem (35) can be constructed as $\overline{\boldsymbol{v}}=\boldsymbol{\Pi} \boldsymbol{\Lambda}^{1 / 2} \boldsymbol{r}$, where $\boldsymbol{r} \sim \mathcal{C N}\left(0, \mathbf{I}_{N}\right)$ is a random vector. The maximum objective function of problem (35) can be obtained by finding the best $\boldsymbol{r}^{*}$. Therefore, we can obtain $\overline{\boldsymbol{v}}^{*}=\boldsymbol{\Pi} \boldsymbol{\Lambda}^{1 / 2} \boldsymbol{r}^{*}$. Since $\overline{\boldsymbol{v}}^{*}$ is the suboptimal solution of problem (35), we have $\boldsymbol{v}^{*}=\exp \left\{j \arg \left(\left[\frac{\overline{\boldsymbol{v}}^{*}}{\overline{\boldsymbol{v}}^{*} N}\right]_{1: N}\right)\right\}$. As a result, the optimal phase-shift matrix is $\boldsymbol{\Theta}^{*}=\operatorname{diag}\left(\boldsymbol{v}^{*}\right)$.

\section{Complexity Analysis}

In this subsection, we analyze the computational complexity of Algorithm 1. Since the maximum inner iteration number is $L_{\max }$, the calculation of $\mathbf{W}^{*}$ requires the eigenvalue decomposition of a $M \times M$ matrix $\mathbf{G}$ with the complexity of $\mathcal{O}\left(L_{\max } M^{3}\right)$ [36]. According to (30), the iteration number

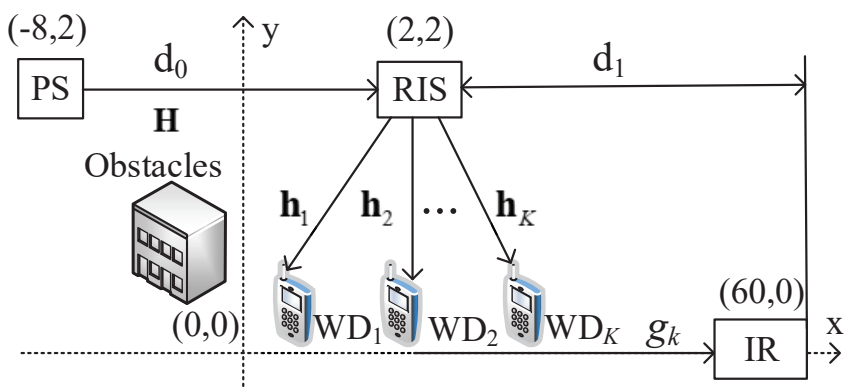

Fig. 4. The simulated network model.

for $p_{k}$ is $\mathcal{O}\left(L_{\max } K\right)$. Based on the subgradient method, the maximum iteration number is $\mathcal{O}\left(L_{\max } K\right)$ according to (26), (31), and (32)-(34). Thus, the complexity of problem (17) is $\mathcal{O}\left(L_{\max } M^{3}\right)$. The SDP problem (36) can be solved with a worst-case complexity of $\mathcal{O}\left\{(N+1)^{4.5}\right\}$ [36]. In summary, the computational complexity of the proposed algorithm is $\mathcal{O}\left\{I_{\max }\left(L_{\max } M^{3}+(N+1)^{4.5}\right)\right\}$.

\section{Simulation Results}

In this section, simulation results are provided to evaluate the effectiveness of the proposed algorithm. The simulated network model is illustrated in Fig. 4. The coordinates of the PS, the RIS and the IR are $(-8,2),(2,2)$, and $(60,0)$, respectively. The WDs are randomly and uniformly distributed within a circular area centered at $(2,0)$ with radius $1 \mathrm{~m}$. The path-loss model is $\Gamma(d)=\Gamma_{0}\left(\frac{d}{d_{\mathrm{R}}}\right)^{-\alpha}$, where $\Gamma_{0} \sim \mathcal{C N}(0,1)$ denotes the path-loss factor at the reference distance $d_{\mathrm{R}}=1$ $\mathrm{m}, d$ is the distance between one transmitter and one receiver, and $\alpha \in[2,5]$ denotes the path-loss exponent [34]-[36]. Other parameters are: $\chi=0.8, \quad P_{k}^{\mathrm{C}}=7 \mathrm{dBm}, P_{e}=1.8 \mathrm{dBm}$, $P^{\mathrm{PS}}=17 \mathrm{dBm}, P^{\mathrm{IR}}=17 \mathrm{dBm}, \delta^{2}=-80 \mathrm{dBm}, P^{\mathrm{max}}=25$ $\mathrm{dBm}, R_{k}^{\min }=0.5$ bits $/ \mathrm{Hz}, I_{\max }=10^{5}, L_{\max }=10^{5}$, $\varepsilon=10^{-5}, d_{0}=10 \mathrm{~m}, d_{1}=60 \mathrm{~m}, T=1 \mathrm{~s}, M=6$, $N=30$, and $K=4$.

\section{A. Performance Evaluation of the Proposed Algorithm}

Fig. 5 shows the total EE of WDs versus the number of iterations. The value of the minimum throughput threshold of each WD is set to $R_{k}^{\min }=0.5 \mathrm{bits} / \mathrm{Hz}$. The proposed algorithm can converge quickly even if the values of $M$ and $N$ are large. Additionally, the total EE of WDs increases with the increasing $N$. The reason is that a large number of reflecting elements can reflect more energy signals to the WDs and increase the harvested energy.

Fig. 6 depicts the total EE of WDs versus the number of antennas of the PS. From the figure, the total EE of WDs improves quickly with the increasing number of antennas $M$. What's more, with the increasing transmit power threshold $P^{\text {max }}$, the total EE of WDs increases accordingly. Since a bigger $P^{\text {max }}$ provides more available energy signals to the WDs so that they can harvest more wireless energy for the data transmission during phase II.

Fig. 7 depicts the total EE of WDs versus the minimum throughput threshold of each WD. The total EE of WDs 


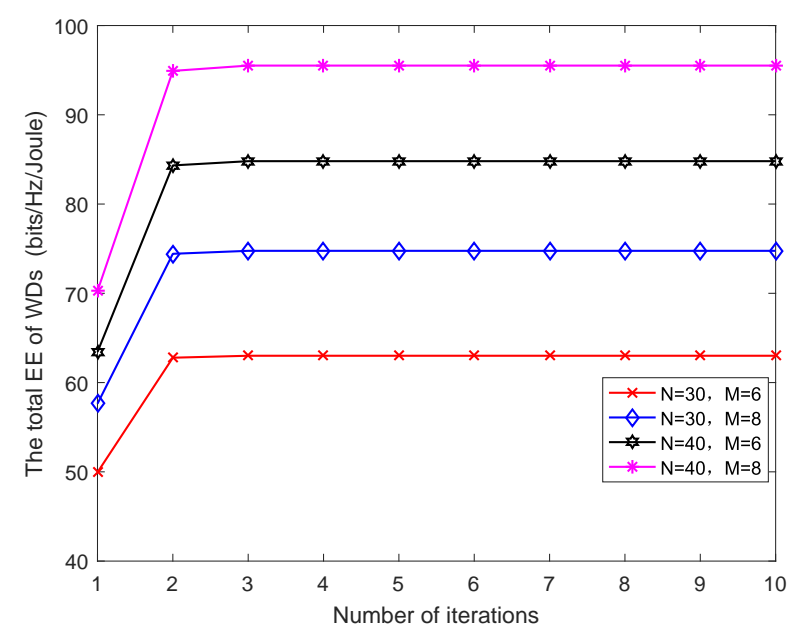

Fig. 5. The convergence of the proposed algorithm.

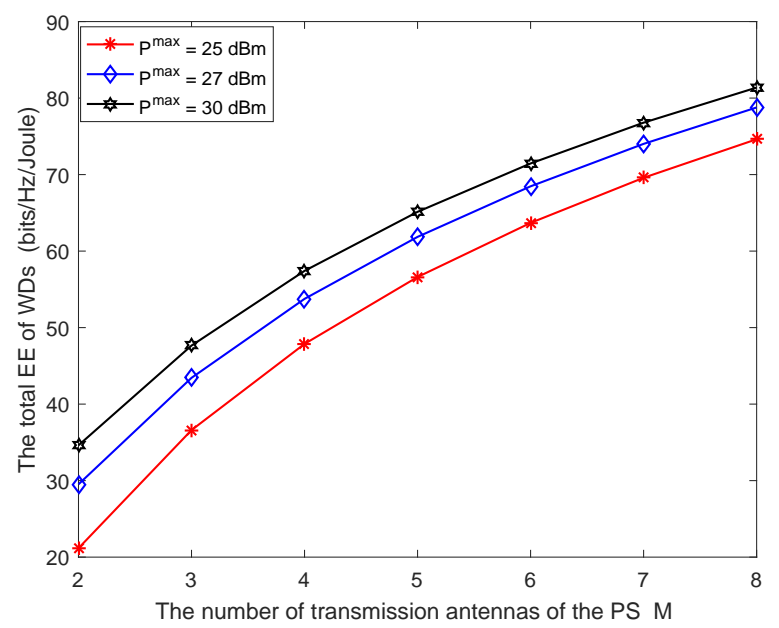

Fig. 6. The total EE of WDs versus the number of antennas of the PS $M$.

decreases with the increasing throughput threshold $R_{k}^{\min }$. Because each WD requires to provide more transmit power for guaranteeing the QoS constraint so that the total power consumption of WDs under phase II increases a lot. Additionally, the improvement of throughput of WDs is smaller than that of total transmit power consumption.

Fig. 8 shows the total EE of WDs versus the number of reflecting elements of the RIS $N$. With the increasing $N$, the total EE of WDs increases quickly and then moderately. Since the harvested energy of WDs increases with the bigger $N$, and the total EE improves a lot accordingly. Moreover, the circuit power consumption of the RIS increases with the increasing number of reflecting elements, which leads to a slowly increasing EE. Furthermore, the total EE under a small $P_{e}$ is larger than that under a big $P_{e}$, because the total EE is a monotonically decreasing function with $P_{e}$.

\section{B. Performance Comparison}

To further demonstrate the effectiveness of the proposed algorithm, we compare the proposed algorithm with different

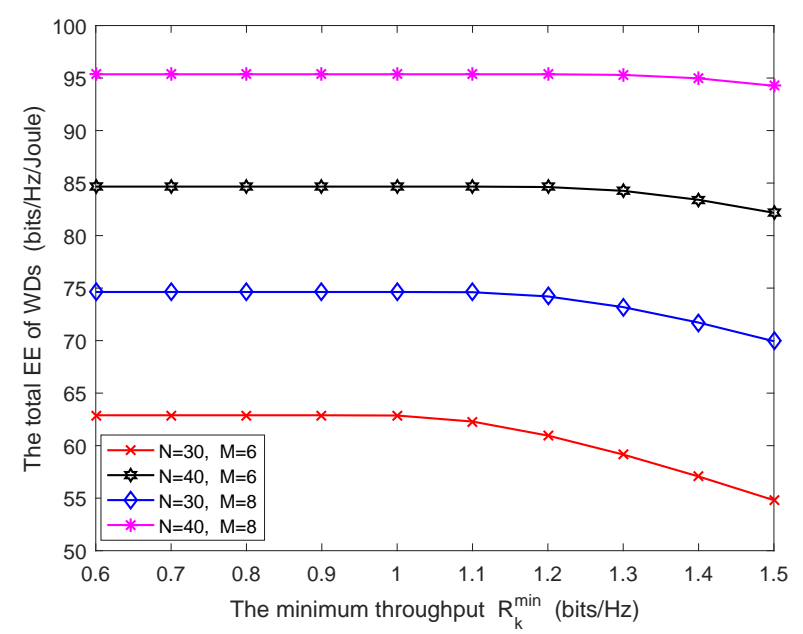

Fig. 7. The total EE of WDs versus the minimum throughput threshold of each WD $R_{k}^{\min }$.

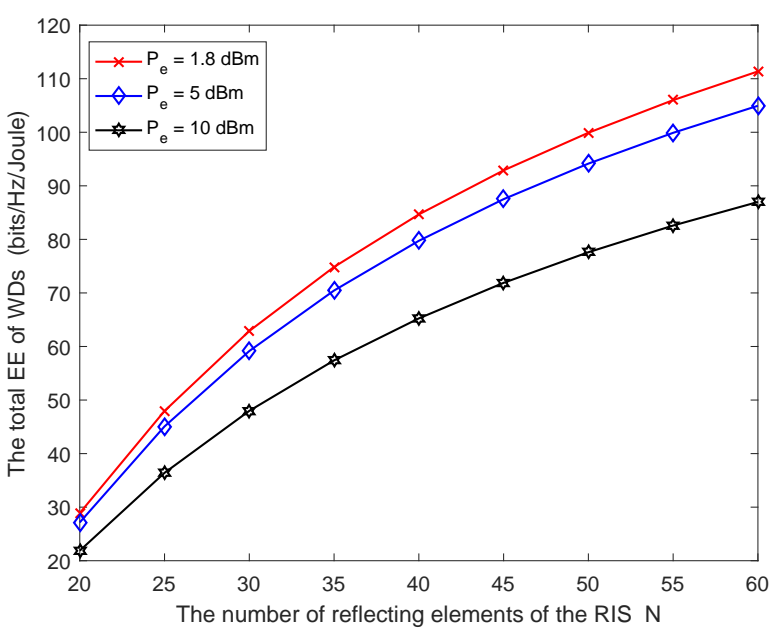

Fig. 8. The total EE of WDs versus the number of reflecting elements of the RIS $N$.

algorithms.

- The baseline algorithm: the EE-based maximization RA algorithm is achieved under random phase shifts.

- The existing algorithm: the throughput-based maximization algorithm is obtained via [36].

Fig. 9 shows the total EE of WDs versus the maximum transmit power of the PS under different algorithms. Obviously, the total EE of WDs under both the proposed algorithm and the baseline algorithm increase with the increase of the transmit power threshold of the PS $P^{\max }$. However, the total EE of the existing algorithm first increases and then decreases (e.g., after $P^{\max }=26 \mathrm{dBm}$ ). The reason is that the existing algorithm consumes more transmit power to improve the sum throughput of the WDs, but ignores the trade-off between the improvement of the total throughput and the total power consumption. Moreover, the total EE of the proposed algorithm is the best one. 


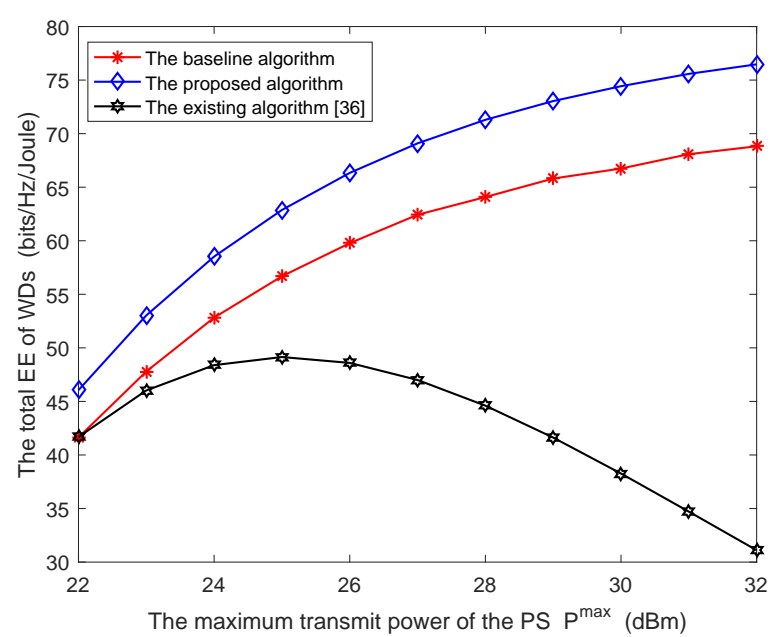

Fig. 9. The total EE of WDs versus the maximum transmit power of the PS $P^{\max }$.

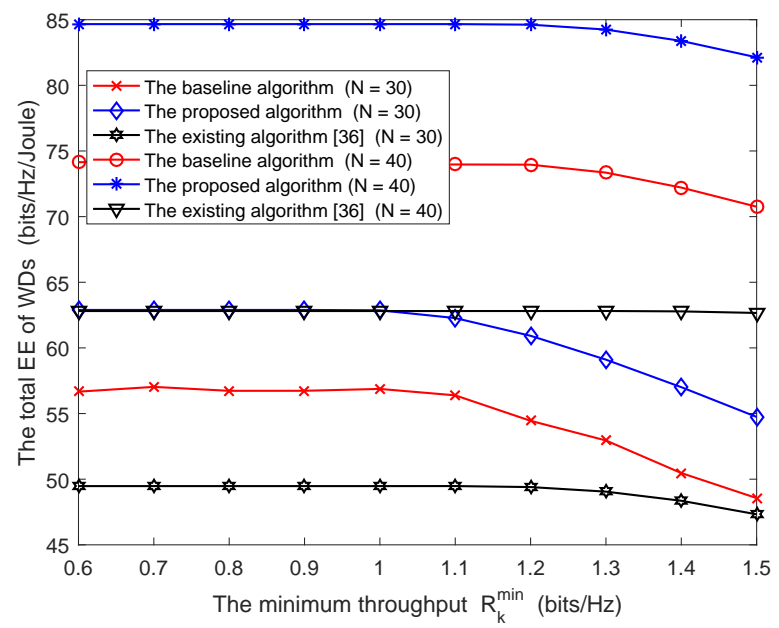

Fig. 10. The total EE of WDs versus the minimum throughput threshold of each WD $R_{k}^{\min }$.

Fig. 10 depicts the total EE of WDs versus the minimum throughput of the WD (i.e., $R_{k}^{\mathrm{min}}$ ) under different algorithms. The number of antennas of the PS is set to $M=6$. It is observed that the total EE of WDs remains unchanged under a small throughput threshold and then decreases under a large throughput threshold. The proposed algorithm has the highest EE. The existing algorithm has the lowest EE. The reason is that, under the small throughput requirement, the throughput constraint is easy to guarantee so that the optimal solution does not change. The total EE remains unchanged. In addition, under a large throughput threshold, the feasible region of the RA problem becomes small so that the total EE of WDs decreases accordingly.

Fig. 11 shows the total EE of WDs versus the distance between the IR and the WDs under different algorithms. It can be seen that with the increasing distance between the IR and the WDs (i.e., $d_{1}$ ), the total EE of WDs decreases a lot. The reason is that, under the same distance between the PS

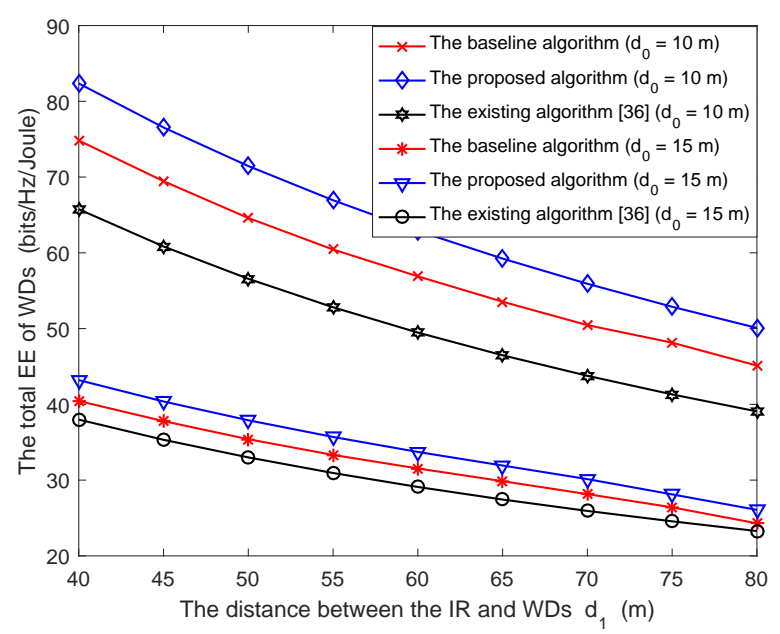

Fig. 11. The total EE of WDs versus the distance between the IR and WDs $d_{1}$.

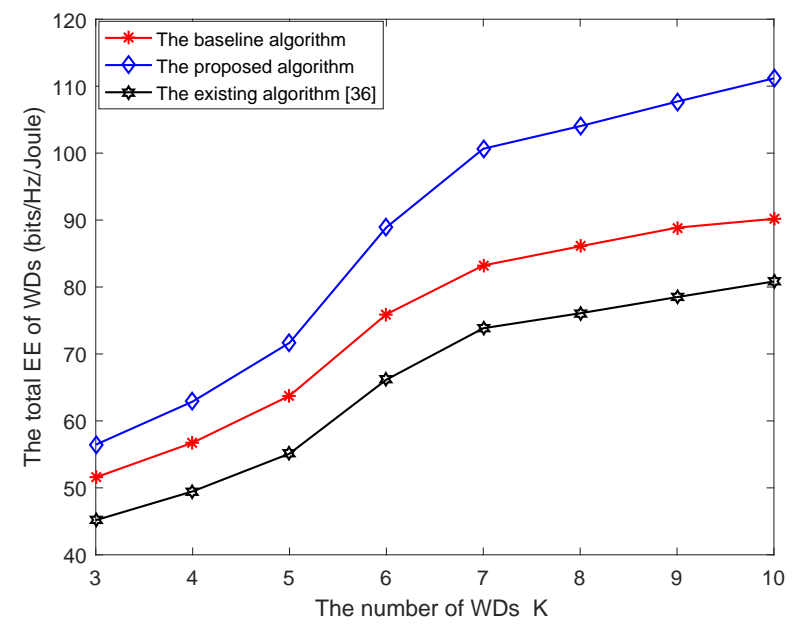

Fig. 12. The total EE of WDs versus the number of WDs $K$.

and the RIS (i.e., $d_{0}$ ), the sum throughput of WDs decreases as the increasing distance from WDs to the IR. Moreover, the transmit power of WDs increases accordingly. As a result, the total EE of WDs decreases. Moreover, the total EE of WDs decreases with the increasing distance between the PS and the RIS (i.e., $d_{0}$ ). Because the bigger $d_{0}$ means that the available energy arrived at the RIS is small so that the harvested energy of WDs becomes small.

Fig. 12 depicts the total EE of WDs versus the number of WDs under different algorithms. With the increasing number of WDs (i.e., $K$ ), the total EE of WDs under different algorithms increases accordingly. The reason is that more wireless energy is harvested under the increasing number of WDs. In addition, the total EE of WDs under the proposed algorithm is much larger than that of the other algorithms.

Fig. 13 shows the total EE of WDs versus the power consumption of each reflecting element under different algorithms. From the figure, the total EE of WDs under different algorithms decreases with the increasing $P_{e}$. Based on the 


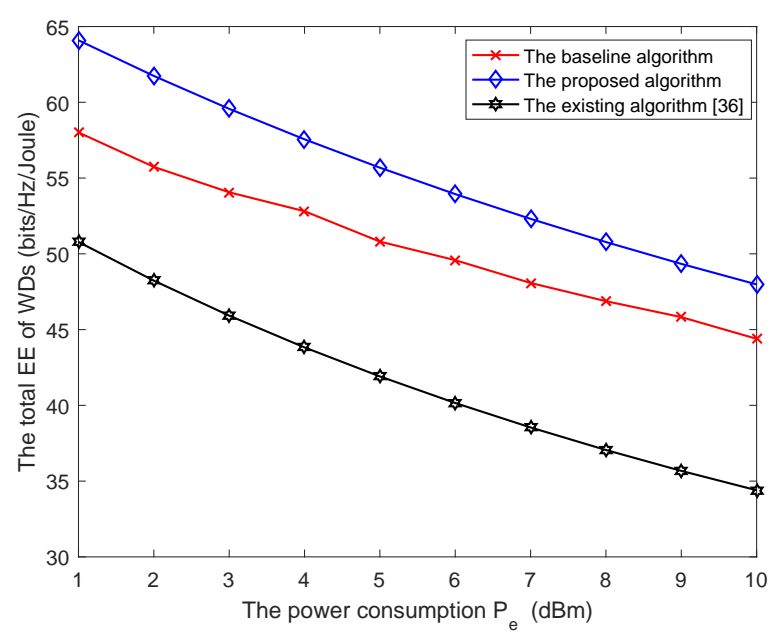

Fig. 13. The total EE of WDs versus the power consumption of each reflecting element $P_{e}$

objective function in problem (13), the total EE is a decreasing function with the circuit power consumption of the RIS. Therefore, it is necessary to consider the impact of circuit power consumption of the RIS on system performance.

\section{CONCLUSIONS}

In this paper, we studied the EE-based maximization problem in a multiuser RIS-assisted WPCN with the EH and minimum throughput constraints of WDs, where the RIS was used to help the WET. We employed a HTT protocol for battery-less WDs to harvest energy in the WET phase and then achieved information transmission in the WIT phase. The EE-based RA problem was formulated by jointly optimizing the energy beamforming of the PS, the passive beamforming of the RIS, the energy-harvesting time, and the transmit power of WDs. According to the problem structure, we converted the original problem into a subtractive form via Dinkelabch's method, and then decomposed the problem into two subproblems that could be solved individually. The simulation results demonstrated the effectiveness of the proposed algorithm compared to the baseline algorithm and the existing algorithm (i.e., the throughputbased maximization algorithm).

\section{APPENDIX A}

\section{THE PROOF OF THEOREM 1}

Since $\overline{\mathbf{G}}=\sum_{k=1}^{K}\left(\eta+\varphi_{k}\right) \chi \mathbf{G}_{k}^{\mathrm{H}} \mathbf{G}_{k}-(\eta+\mu) \mathbf{I}_{M}$ and $\mathbf{G}=$ $\sum_{k=1}^{K}\left(\eta+\varphi_{k}\right) \chi \mathbf{G}_{k}^{\mathrm{H}} \mathbf{G}_{k}$, we have $\overline{\mathbf{G}}=\mathbf{G}-(\eta+\mu) \mathbf{I}_{M}$. Based on (22) i.e.,

$L\left(\overline{\mathbf{W}}, t_{0}, \boldsymbol{t}, \overline{\boldsymbol{p}}, \boldsymbol{\varphi}, \boldsymbol{\beta}, \kappa, \mu\right)=\bar{L}\left(t_{0}, \boldsymbol{t}, \overline{\boldsymbol{p}}, \boldsymbol{\varphi}, \boldsymbol{\beta}, \kappa, \mu\right)+\operatorname{Tr}(\overline{\mathbf{G}} \overline{\mathbf{W}})$,

According to KKT conditions [45], the optimal $\overline{\mathbf{W}}^{*}$ satisfies

$$
\begin{gathered}
\overline{\mathbf{G}} \overline{\mathbf{W}}^{*}=\mathbf{0}, \overline{\mathbf{W}}^{*} \succeq \mathbf{0}, \\
\varphi_{k}\left\{\eta\left(\mathbf{G}_{k} \overline{\mathbf{W}}^{*} \mathbf{G}_{k}^{\mathrm{H}}\right)-\left(\bar{p}_{k}+P_{k}^{\mathrm{C}}\left(t_{0}+t_{k}\right)\right)\right\}=0,
\end{gathered}
$$

$$
\mu\left\{t_{0} P^{\max }-\operatorname{Tr}\left(\overline{\mathbf{W}}^{*}\right)\right\}=0 .
$$

Since $\overline{\mathbf{W}} \succeq \mathbf{0}$, we have $\operatorname{Tr}(\overline{\mathbf{G}} \overline{\mathbf{W}}) \leq 0$. There is

$$
\operatorname{Tr}\left(\overline{\mathbf{G}} \overline{\mathbf{W}}^{*}\right)=0 \Leftrightarrow \overline{\mathbf{G}} \overline{\mathbf{W}}^{*}=\mathbf{0} .
$$

Since $\eta \geq 0, \varphi_{k} \geq 0$, and $\mathbf{G} \succeq \mathbf{0}, \mathbf{G}$ can be decomposed as $\mathbf{G}=\mathbf{U} \sum \mathbf{U}^{\mathrm{H}}$, where $\mathbf{U} \in \mathbb{C}^{M \times M}$ and $\sum=\operatorname{diag}\left(\sigma_{1}, \sigma_{2}, \cdots, \sigma_{\mathrm{M}}\right)$ are the corresponding eigenvector matrix and eigenvalue matrix, respectively. Thus, we have $\overline{\mathbf{G}}=\mathbf{U}\left(\sum-(\eta+\mu) \mathbf{I}_{M}\right) \mathbf{U}^{\mathrm{H}}$, where $\sum-(\eta+\mu) \mathbf{I}_{M}=$ $\operatorname{diag}\left(\sigma_{1}-(\eta+\mu), \sigma_{2}-(\eta+\mu), \cdots, \sigma_{M}-(\eta+\mu)\right)$ is an eigenvalue matrix of $\overline{\mathbf{G}}$. $\mathbf{U} \in \mathbb{C}^{M \times M}$ is the eigenvector matrix of $\overline{\mathbf{G}}$. Just to make sure that $\overline{\mathbf{G}}$ is a negative semidefinite matrix, $\eta+\mu^{*}=\sigma_{1}>0$ holds under any $\eta$ and $\mu^{*}$. Based on $\overline{\mathbf{G}} \mathbf{u}_{1}=\mathbf{0}$ and $\overline{\mathbf{G}} \overline{\mathbf{W}}^{*}=\mathbf{0}$, the optimal $\overline{\mathbf{W}}^{*}$ is

$$
\overline{\mathbf{W}}^{*}=\rho \mathbf{u}_{1} \mathbf{u}_{1}^{\mathrm{H}},
$$

where $\rho \geq 0$. Based on $\operatorname{Tr}\left(\overline{\mathbf{W}}^{*}\right)=t_{0} P^{\max }$ and $\operatorname{Tr}\left(\overline{\mathbf{W}}^{*}\right)=$ $\rho$, we have $\rho=t_{0} P^{\max }$ and $\overline{\mathbf{W}}^{*}=t_{0} P^{\max } \mathbf{u}_{1} \mathbf{u}_{1}^{\mathrm{H}}$.

The proof is complete.

\section{REFERENCES}

[1] L. Chettri and R. Bera, "A comprehensive survey on Internet of things (IoT) toward 5G wireless systems," IEEE Internet Things J., vol. 7, no. 1, pp. 16-32, Jan. 2020.

[2] Y. Xu, G. Gui, H. Gacanin and F. Adachi, "A survey on resource allocation for 5G heterogeneous networks: Current research, future trends, and challenges," IEEE Commun. Surveys Tuts. vol. 23, no. 2 pp. 668-695, 2nd Quart. 2021.

[3] Y. Xu, X. Zhao and Y. Liang, "Robust power control and beamforming in cognitive radio networks: A survey," IEEE Commun. Surveys Tuts., vol. 17, no. 4, pp. 1834-1857, 4th Quart. 2015.

[4] I. Abu Mahady, E. Bedeer, S. Ikki and H. Yanikomeroglu, "Sumrate maximization of NOMA systems under imperfect successive interference cancellation," IEEE Commun. Lett., vol. 23, no. 3, pp. 474-477, Mar. 2019.

[5] X. Lu, P. Wang, D. Niyato, D. I. Kim and Z. Han, "Wireless networks with RF energy harvesting: A contemporary survey," IEEE Commun. Surveys Tuts., vol. 17, no. 2, pp. 757-789, 2nd Quart. 2015.

[6] S. Bi, Y. Zeng and R. Zhang, "Wireless powered communication networks: An overview," IEEE Wireless Commun., vol. 23, no. 2, pp. 10-18, Apr. 2016.

[7] J. Kim, H. Lee, C. Song, T. Oh and I. Lee, "Sum throughput maximization for multi-user MIMO cognitive wireless powered communication networks," IEEE Trans. Wireless Commun., vol. 16, no. 2, pp. 913-923, Feb. 2017.

[8] I. Krikidis, "Average age of information in wireless powered sensor networks," IEEE Wireless Commun. Lett., vol. 8, no. 2, pp. 628-631, Apr. 2019.

[9] A. Asadi, Q. Wang and V. Mancuso, "A survey on device-to-device communication in cellular networks," IEEE Commun. Surveys Tuts. vol. 16, no. 4, pp. 1801-1819, 4th Quart. 2014.

[10] S. Movassaghi, M. Abolhasan, J. Lipman, D. Smith and A. Jamalipour, "Wireless body area networks: A survey," IEEE Commun. Surveys Tuts., vol. 16, no. 3, pp. 1658-1686, 3rd Quart. 2014.

[11] H. Ju and R. Zhang, "Throughput maximization in wireless powered communication networks," IEEE Trans. Wireless Commun., vol. 13, no. 1, pp. 418-428, Jan. 2014

[12] B. Lyu, T. Qi, H. Guo and Z. Yang, "Throughput maximization in fullduplex dual-hop wireless powered communication networks," IEEE Access, vol. 7, pp. 158584-158593, 2019.

[13] S. Lohani, R. A. Loodaricheh, E. Hossain and V. K. Bhargava, "On multiuser resource allocation in relay-based wireless-powered uplink cellular networks," IEEE Trans. Wireless Commun., vol. 15, no. 3, pp. 1851-1865, Mar. 2016.

[14] L. Xie, J. Xu and R. Zhang, "Throughput maximization for UAVenabled wireless powered communication networks," IEEE Internet Things J., vol. 6, no. 2, pp. 1690-1703, Apr. 2019. 
[15] W. Wang, J. Tang, N. Zhao, X. Liu, X. Zhang, Y. Chen and Y. Qian, "Joint precoding optimization for secure SWIPT in UAV-aided NOMA networks," IEEE Trans. Commun., vol. 68, no. 8, pp. 5028-5040, Aug. 2020.

[16] X. Pang, J. Tang, N. Zhao, X. Zhang and Y. Qian, "Energy-efficient design for mmWave-enabled NOMA-UAV networks," Sci. China Inf. Sci., vol. 64, no. 4, pp. 1403033, 2021.

[17] G. Yang, D. Yuan, Y. Liang, R. Zhang and V. C. M. Leung, "Optimal resource allocation in full-duplex ambient backscatter communication networks for wireless-powered IoT," IEEE Internet Things J., vol. 6, no. 2, pp. 2612-2625, Apr. 2019.

[18] S. Lee and R. Zhang, "Cognitive wireless powered network: Spectrum sharing models and throughput maximization," IEEE Trans. Cog. Commun. Netw., vol. 1, no. 3, pp. 335-346, Sept. 2015.

[19] H. Lee, K. Lee, H. Kim, B. Clerckx and I. Lee, "Resource allocation techniques for wireless powered communication networks with energy storage constraint," IEEE Trans. Wireless Commun., vol. 15, no. 4, pp. 2619-2628, Apr. 2016

[20] Q. Sun, G. Zhu, C. Shen, X. Li and Z. Zhong, "Joint beamforming design and time allocation for wireless powered communication networks," IEEE Commun. Lett., vol. 18, no. 10, pp. 1783-1786, Oct 2014

[21] L. Liu, R. Zhang and K. Chua, "Multi-antenna wireless powered communication with energy beamforming," IEEE Trans. Commun., vol. 62, no. 12, pp. 4349-4361, Dec. 2014.

[22] H. Ju and R. Zhang, "User cooperation in wireless powered communication networks," in Proc. IEEE GLOBECOM, Austin, TX, USA 2014, pp. 1430-1435.

[23] Z. Chu, F. Zhou, Z. Zhu, M. Sun and N. Al-Dhahir, "Energy beamforming design and user cooperation for wireless powered communication networks," IEEE Wireless Commun. Lett., vol. 6, no. 6, pp. 750-753, Dec. 2017

[24] Z. Chang, Z. Wang, X. Guo, Z. Han and T. Ristaniemi, "Energyefficient resource allocation for wireless powered massive MIMO system with imperfect CSI," IEEE Trans. Green Commun. Netw., vol. 1, no. 2, pp. 121-130, Jun. 2017.

[25] Q. Wu, M. Tao, D. W. Kwan Ng, W. Chen and R. Schober, "Energyefficient resource allocation for wireless powered communication networks," IEEE Trans. Wireless Commun., vol. 15, no. 3, pp. 2312-2327, Mar. 2016.

[26] C. Huang, R. Mo and C. Yuen, "Reconfigurable intelligent surface assisted multiuser MISO systems exploiting deep reinforcement learning," IEEE J. Sel. Areas Commun., vol. 38, no. 8, pp. 1839-1850, Aug. 2020

[27] E. Basar, M. Di Renzo, J. De Rosny, M. Debbah, M. -S. Alouini and R. Zhang, "Wireless communications through reconfigurable intelligent surfaces," IEEE Access, vol. 7, pp. 116753-116773, 2019.

[28] Z. Wang, Y. Shi, Y. Zhou, H. Zhou and N. Zhang, "Wireless-powered over-the-air computation in intelligent reflecting surface-aided IoT networks," IEEE Internet Things J., vol. 8, no. 3, pp. 1585-1598, Feb. 2021.

[29] C. Huang, A. Zappone, G. C. Alexandropoulos, M. Debbah and C. Yuen, "Reconfigurable intelligent surfaces for energy efficiency in wireless communication," IEEE Trans. Wireless Commun., vol. 18, no. 8, pp. 4157-4170, Aug. 2019.

[30] Q. Wu and R. Zhang, "Joint active and passive beamforming optimization for intelligent reflecting surface assisted SWIPT under QoS constraints," IEEE J. Sel. Areas Commun., vol. 38, no. 8, pp. 17351748, Aug. 2020

[31] M. A. ElMossallamy, H. Zhang, L. Song, K. G. Seddik, Z. Han and G. Y. Li, "Reconfigurable intelligent surfaces for wireless communications: Principles, challenges, and opportunities," IEEE Trans. Cog. Commun. Netw., vol. 6, no. 3, pp. 990-1002, Sept. 2020.

[32] B. Di, H. Zhang, L. Song, Y. Li, Z. Han and H. V. Poor, "Hybrid beamforming for reconfigurable intelligent surface based multi-user communications: Achievable rates with limited discrete phase shifts," IEEE J. Sel. Areas Commun., vol. 38, no. 8, pp. 1809-1822, Aug. 2020

[33] H. Zhang, B. Di, L. Song and Z. Han, "Reconfigurable intelligent surfaces assisted communications with limited phase shifts: How many phase shifts are enough?," IEEE Trans. Veh. Technol., vol. 69, no. 4 pp. 4498-4502, Apr. 2020.

[34] B. Lyu, D. T. Hoang, S. Gong and Z. Yang, "Intelligent reflecting surface assisted wireless powered communication networks," in Proc. WCNC Workshops, Seoul, Korea (South), 2020, pp. 1-6.

[35] Y. Zheng, S. Bi, Y. J. Zhang, Z. Quan and H. Wang, "Intelligent reflecting surface enhanced user cooperation in wireless powered communication networks," IEEE Wireless Commun. Lett., vol. 9, no. 6, pp. 901-905, Jun. 2020

[36] Y. Zheng, S. Bi, Y. -J. A. Zhang, X. Lin and H. Wang, "Joint beamforming and power control for throughput maximization in IRSassisted MISO WPCNs," IEEE Internet Things J., vol. 8, no. 10, pp 8399-8410, May 2021.

[37] B. Lyu, P. Ramezani, D. T. Hoang, S. Gong, Z. Yang and A. Jamalipour, "Optimized energy and information relaying in self-sustainable IRS empowered WPCN," IEEE Trans. Commun., vol. 69, no. 1, pp. 619 633, Jan. 2021

[38] F. E. Bouanani, S. Muhaidat, P. C. Sofotasios, O. A. Dobre and O. S. Badarneh, "Performance analysis of intelligent reflecting surface aided wireless networks with wireless power transfer," IEEE Commun. Lett. vol. 25, no. 3, pp. 793-797, Mar. 2021

[39] J. Lee and K. Lee, "Effects of number of relays on achievable efficiency of magnetic resonant wireless power transfer," IEEE Trans. Power Electron., vol. 35, no.7, pp. 6697-6700, Jul. 2020.

[40] S. Pejoski, Z. Hadzi-Velkov and R. Schober, "Optimal power and time allocation for WPCNs with piece-wise linear EH model," IEEE Wireless Commun. Lett., vol. 7, no. 3, pp. 364-367, Jun. 2018.

[41] E. Boshkovska, D. W. K. Ng, N. Zlatanov, A. Koelpin and R. Schober, "Robust resource allocation for MIMO wireless powered communication networks based on a non-linear EH model," IEEE Trans. Commun. vol. 65, no. 5, pp. 1984-1999, May 2017.

[42] W. Dinkelbach, "On nonlinear fractional programming," Manage. Sci. vol. 13, no. 7, Mar. 1967

[43] Z. Yang, J. Shi, Z. Li, M. Chen, W. Xu and M. Shikh-Bahaei, "Energy efficient rate splitting multiple access (RSMA) with reconfigurable intelligent surface," in Proc. IEEE ICC Workshops, Dublin, Ireland, 2020, pp. 1-6.

[44] $\mathrm{Y}, \mathrm{Xu}, \mathrm{R}, \mathrm{Q}, \mathrm{Hu}$ and $\mathrm{Y}, \mathrm{Qian}$ "Robust max-min fairness energy efficiency in NOMA-based heterogeneous networks," in Proc. IEEE ICC, Dublin, Ireland, 2020, pp. 1-6.

[45] S. Boyd and L. Vandenberghe, Convex Optimization. Cambridge, U.K. Cambridge Univ. Press, 2004

[46] Z. -Q. Luo, W. K. Ma, A. M. C. So, Y. Ye and S. Zhang, "Semidefinite relaxation of quadratic optimization problems," IEEE Signal Process. Mag., vol. 27, no. 3, pp. 20-34, May 2010.

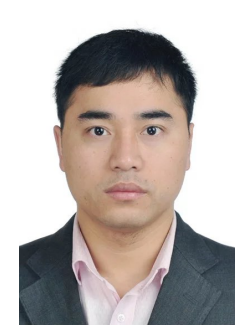

Yongjun Xu (Senior Member, IEEE) received the Ph.D. degree (Hons.) in communication and information system from Jilin University, Changchun, China, in 2015. He received the Outstanding Doctoral Thesis of Jilin Province in 2016. He is currently an Associate Professor with the Schoo of Communication and Information Engineering, Chongqing University of Posts and Telecommunications, Chongqing, China. From 2018 to 2019, he was a Visiting Scholar with Utah State University, Logan, UT, USA. He has authored or coauthored more than 80 papers, including the IEEE Communications Surveys \& Tutorials, IEEE Transactions on Vehicular Technology, IEEE Transactions on Cognitive Communications and Networking, IEEE Transactions on Green Communications and Networking, IEEE Internet of Tings Journal, IEEE Communications Letters, IEEE Wireless Communications Letters, etc.

His recent interests include heterogeneous networks, resource allocation, reconfigurable intelligent surface, energy harvesting, backscatter communications, mobile edge computing, UAV communications. He serves as an Associate Editor of EURASIP Journal on Wireless Communications and Networking, an Editor of Physical Communications, an Academic Editor of Digital Communications and Networks. He serves as a reviewer for IET Communications, IEEE Transactions on Wireless Communications, IEEE Transactions on Vehicular Technology, IEEE Communications Letters, IEEE Wireless Communications Letters, and China Communications. He was the Technical Program Committee member for many international conferences, such as GLOBECOM, ICC, ICCT, WCNC, ICCC, and CITS. 


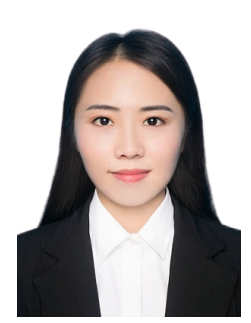

Zhengnian Gao received the B.Eng. degree from the University of South China, Hunan, China, in 2019. She is currently pursuing the M.S. degree with the Chongqing University of Posts and Telecommunications, Chongqing, China. Her current research interests include energy harvesting, reconfigurable intelligent surface, and resource allocation.

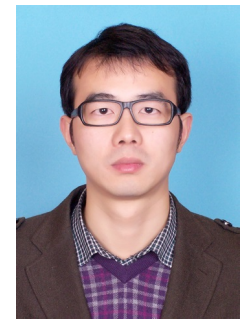

Zhengqiang Wang (Member, IEEE) received the B.S. degree from southeast University, Nanjing, China, in 2005, and the M.S. degree from Xuzhou Normal University, Xuzhou, China, in 2008, all in applied mathematics, and the Ph.D. degree from the Department of Electronic Engineering, Shanghai Jiao Tong University, China, in 2015. He is currently an Associate Professor with the School of Communications and Information Engineering, Chongqing University of Posts and Telecommunications. His current research interests include green networks, massive MIMO, NOMA, UAV communication, game theory, and network optimization.

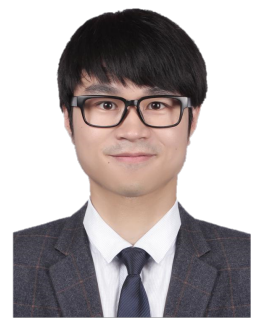

Zhaohui Yang (Member, IEEE) received the B.S degree in information science and engineering from Chien-Shiung Wu Honors College, Southeast University, Nanjing, China, in June 2014, and the Ph.D. degree in communication and information system with the National Mobile Communications Research Laboratory, Southeast University, Nanjing, China, in May 2018. From May 2018 to October 2020, he was a postdoctoral research associate with the Center for Telecommunications Research, Department of Informatics, King's College London, UK.

$\mathrm{He}$ is currently a visiting associate professor with College of Information Science and Electronic Engineering Zhejiang Key Lab of Information Processing Communication and Networking, Zhejiang University, and also a research fellow with the Department of Electronic and Electrical Engineering, University College London, UK. He is an Associate Editor for the IEEE Communications Letters, IET Communications and EURASIP Journal on Wireless Communications and Networking. He has guest edited a feature topic of IEEE Communications Magazine on Communication Technologies for Efficient Edge Learning. He was a Co-Chair for workshops on edge learning and wireless communications in several conferences including the IEEE International Conference on Communications (ICC), the IEEE Global Telecommunication Conference (GLOBECOM), the IEEE Wireless Communications and Networking Conference (WCNC), and the IEEE International Symposium on Personal, Indoor and Mobile Radio Communication (PIMRC). His research interests include federated learning, reconfigurable intelligent surface, UAV, and NOMA. He was a TPC member of IEEE ICC during 20152021 and GLOBECOM during 2017-2021. He was an exemplary reviewer for IEEE Transactions on Communications in 2019 and 2020

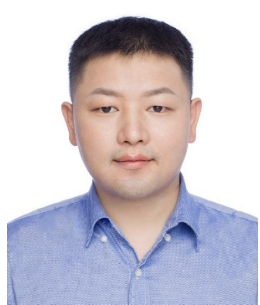

Chongwen Huang (Member, IEEE) obtained his B Sc. degree in 2010 from Nankai University, Binhai College, and the M.Sc degree from the University of Electronic Science and Technology of China (UESTC, Chengdu) in 2013. He joined the Institute of Electronics, Chinese Academy of Sciences (IECAS, Beijing) as a research engineer from Jul. 2013. Since Sep. 2015, he started his Ph.D. journey in Singapore University of Technology and Design (SUTD, Singapore) and CentraleSupélec University (Paris, France), under the supervision of Prof. Chau YUEN and Prof. Mérouane DEBBAH. From Oct. 2019 to Sep. 2020, He is a Postdoc in his mother University SUTD. Since Sep. 2020, he joined into Zhejiang University as a tenure-track young professor.

Dr. Huang is a recipient of IEEE Marconi Prize Paper Award in Wireless Communications in 2021. He was also a recipient of Singapore Government Ph.D. scholarship, and received PHC Merlion Ph.D. Grant 2016-2019 for studying in CentraleSupélec, France. He has served as an Editor of Elsevier Signal Processing, EURASIP Journal on Wireless Communications and Networking and Physical Communication since 2021. In addition, Dr. Huang also has served the chair of some wirless communications flagship conference, including session chair of 2021IEEE WCNC, 2021IEEE VTC-Fall, and symposium chair of IEEE WCSP 2021, etc. His main research interests are focused on Holographic MIMO Surface/Reconfigurable Intelligent Surface, B5G/6G Wireless Communication, mmWave/THz Communications, Deep Learning technologies for Wireless communications, etc.

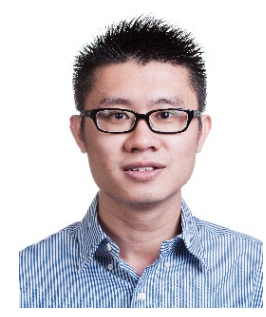

Chau Yuen (S'02-M'06-SM'12-F'21) received the B.Eng. and Ph.D. degrees from Nanyang Technological University (NTU), Singapore, in 2000 and 2004, respectively. He was a Post-Doctoral Fellow with Lucent Technologies Bell Labs, Murray Hill, in 2005, and a Visiting Assistant Professor with The Hong Kong Polytechnic University in 2008. From 2006 to 2010, he was with the Institute for Infocomm Research (I2R), Singapore. Since 2010, he has been with the Singapore University of Technology and Design. Dr. Yuen was a recipient of the Lee Kuan Yew Gold Medal, the Institution of Electrical Engineers Book Prize, the Institute of Engineering of Singapore Gold Medal, the Merck Sharp and Dohme Gold Medal, and twice a recipient of the Hewlett Packard Prize. He received the IEEE Asia Pacific Outstanding Young Researcher Award in 2012 and IEEE VTS Singapore Chapter Outstanding Service Award on 2019. He serves as an Editor for the IEEE TRANSACTIONS ON COMMUNICATIONS, and the IEEE TRANSACTIONS ON VEHICULAR TECHNOLOGY, where he was awarded as the Top Associate Editor from 2009 to 2015. He served as the guest editor for several special issues, including IEEE JOURNAL ON SELECTED AREAS IN COMMUNICATIONS, IEEE COMMUNICATIONS MAGAZINE, IEEE TRANSACTIONS ON COGNITIVE COMMUNICATIONS AND NETWORKING. He is a Distinguished Lecturer of IEEE Vehicular Technology Society. 